\title{
Juristenausbildung in der Kritik - Betroffene berichten
}

\author{
Sozialisation eines »neuen Juristen «?
}

Risikovermeidung sollte kein Charakteristikum des neuen Juristen ${ }^{\mathrm{I}}$ sein. Gegenüber der erdrückenden Definitions- und Verhinderungsmacht der sozialen und politischen Umwelt eines jungen juristischen Fachbereiches bleibt aber der Sozialisationserfolg eines mutigen, zu "partielle(r), antizipative(r), folgenreiche(r) Gesellschaftsreform ${ }^{2}{ }^{2}$ gerüsteten Juristen aus. Diesen Sozialisationserfolg hatten sich die Reformer (nicht Revolutionäre) ${ }^{3}$ von reformorientierter Besetzung des Hochschulpersonals, von curricularer Neustrukturierung des Studienganges und der Integration theoretischen Reflexions- und Orientierungswissens in die juristische Ausbildung erhofft.

In der einstufigen Juristenausbildung an der Universität Hannover, von der hier berichtet wird, reduziert sich die Vermittlung solchen allgemeinen Orientierungswissens auf eine zweisemestrige Eingangsphase, innerhalb derer unterschiedliche sozialisations-, klassen-, schicht-, wirtschafts- und staatstheoretische Versatzstücke, abgetrennt vom »eigentlichen« juristischen Studium, die Prämissen juristischer Tätigkeit bezeichnen sollen. Trotz der Inkohärenz und anscheinenden Beliebigkeit, mit der die verschiedensten Theorieansätze vorgestellt werden, hat diese Spielwiese der metajuristischen Betrachtungen im Rahmen des Studiengangs eine einzigartige Bedeutung für jene produktive Verunsicherung des neuen Juristen, die jedem substantiellen Lernen vorausgeht. Einzig hier wird der bornierte Bezugsrahmen juristischer Fachausbildung durchbrochen, die Anpassung an scheinbar unpolitische juristische Entscheidungstechniken und -kriterien wie die simple Identifikation mit Berufsrollen und -moralen kritisiert und konterkariert. Selten habe ich nach dieser ersten Phase meines rechtswissenschaftlichen Studiums wieder derart offene und wirklich kontroverse Diskussionen erlebt. Anders als im harten juristischen Teil des Studiums sind echte Alternativen vertretbar, es kann alles ganz anders sein als von dieser oder jener Sozialisationstheorie vertreten wird. Das vermittelte Orientierungsund Reflexionswissen problematisiert die von den Studenten in die Universität hineingebrachten Einstellungen, Uberzeugungen und Erfahrungen in einer Weise, wie die juristische Fachausbildung dies niemals vermöchte. Juristisches Wissen weist einen derartigen Generalisierungsgrad auf, daß alle Subjektivität derjenigen, die es einüben und anwenden, dahinter verschwindet und jedenfalls auf der bewußten Ebene unthematisiert bleibt. Das ist bei den in der Eingangsphase des Studiums

I Rudolf Wassermann, Theo Rasehorn, Frank Benseler ( $\mathrm{Hg}$.), Der neue Jurist, Materialien zur reformierten Juristenausbildung in Bremen, Reihe Demokratie und Rechtsstaat, Bd. 22, Darmstadt/Neuwied I973.

2 Rudolf Wiethölter, Zur politischen Einschätzung der Einstufenmodelle und -versuche, in: Rudolf Wassermann u. a. (Hg.), Der neue Jurist, a. a. O., S. 232.

3 Thilo Ramm, Reform und keine Revolution, in: Neue Juristenausbildung, Materialien des Loccumer Arbeitskreises, Neuwied/Berlin 1970, S. 77. 
diskutierten, nicht-dogmatisierten "Wirklichkeitswissenschaften « anders. ${ }^{4}$ Wie spannend die Diskussion eines Marx- oder Freud-Textes innerhalb einer Kursgruppe sein kann, wenn diese sich ganz heterogen aus Kommilitonen zusammensetzt, die teils gerade vom Gymnasium, teils vom zweiten Bildungsweg kommen, teilweise bereits im gehobenen Dienst einer Kommunal- oder Bezirksverwaltung bzw. der Polizei Karriere gemacht haben und diese nun durch ein Studium auszubauen trachten, bedarf keiner Phantasie. Hier prallen noch Erfahrungen, Einstellungen und VorUrteile gleichsam roh und ungezügelt aufeinander und werden nicht durch den schnellen Verweis auf Gesatztes, Gesetz und Rechtsprechung im Elan gebremst und in der Sache abgeschnitten, weil rechtlich zwangs $\gg$ rationalisiert «.

Nach der Eingangsphase bekommen dann aber die Juristen, und namentlich deren Eingefleischteste, die Zivil- und Strafrechtler das Sagen. Für dieses Phänomen ist am Fachbereich die Bezeichnung Dogmatikschock geläufig geworden. In der Tat bedeutet der Ubergang in das sogenannte Hauptstudium einen radikalen Bruch in Form und Gegenstand der Ausbildung. Die Vermittlung materiell- und prozeßrechtlicher Kenntnisse bis zum fünften Semester - der hannoversche Studiengang ist ein starres Baukastensystem mit Regelstudienzeit - steht für Studenten wie Lehrende unter zwei ablaufbestimmenden Prämissen. Die Ausbildung dient zum einen unmittelbar der Vorbereitung der referendariatsähnlichen Praktika bei der Zivilgerichtsbarkeit und der obligatorischen Staatsanwaltschaft, zum anderen ist sie an ein geradezu ausgeklügeltes Leistungskontrollsystem gekoppelt.

Der Reformanspruch, alle juristischen Veranstaltungen in die Pflicht zur Integration der Sozialwissenschaften zu nehmen', geht angesichts dieser Prämissen unter. Die Fülle des bis zur Bewährung in der Praxis zu behandlenden Wissensstoffes läßt allein schon die Diskussion von Rechtsproblemen als Luxus erscheinen. Praxis- und klausurrelevante Kurse sind überfüllt, andere wie die arbeitsrechtlichen Angebote werden vernachlässigt. In den überfüllten Kursen wird vorgelesen, einige nehmen den Charakter von Repetitorien $a^{6}{ }^{6}$, verlieren selbst noch das universitäre Gewand. Wie in einem juristischen Studium unvermeidlich, nur eben im Eilverfahren, werden die Systematik von Straftatbeständen, Leistungsstörungen, Eigentumserwerbungen oder der Ablauf eines staatsanwaltschaftlichen Ermittlungsverfahrens unter Einbezug jenes Bestandes an Kasuistik und Dogmatik vermittelt, dessenthalben die Juristen den Theologen gleich ihr Geschäft als Wissenschaft bezeichnet wissen wollen. Sozialwissenschaftlichen Erkenntnissen wird jede praktische Relevanz abgesprochen, im

4 Die wesentliche Stoßrichtung der rechten Kritik an der Einphasenausbildung wendet sich denn auch gegen die Integration der Sozialwissenschaften. Eine sozialwissenschaftliche Ausbildung der Juristen kann nach Helmut Schelsky, Die juridische Rationalität, in: ders., Die Soziologen und das Recht, Opladen 1980, S. 34-76 (68), nur den Sinn haben, xihnen ihre Urteilsunfähigkeit für die politischen, soziologischen, ökonomischen, psychologischen und psychiatrischen usw. Gesichtspunkte des sozialen Zusammenlebens und der ihnen vorliegenden Materien gerichtlicher Urteile oder der Gesetzgebung zu verdeurlichen « und sie so zur bornierten Erledigung ihrer eigentlichen, rein-juridischen Aufgaben zu veranlassen. Die von Scheisky in dieser scheinbar unpolitischen rein-juridischen Tätigkeit ausgemachte institutionelle juridische Rationalität ist nicht mehr an der Bemühung um Wahrheit und Gerechtigkeit orientiert, sondern an der Sicherheit und Gewißheit des institutionellen Status-Quo. Zur Gewährleistung dieser juridischen Rationalität schlägt Schelsky u. a. statt einer sozialwissenschaftlichen Ausbildung vor, die Vermittlung der Gesetzeskenntnisse und dogmatischen Wissensbestände ganz der Repetitorausbildung zu überlassen, »die dies heute bereits meistens besser leistet ... « (S. 70). Engagierte Sozialwissenschaft ist Schelsky ohnehin sozialreligiöse Heilsverkündung, engagierte Rechtssoziologie gar stendenzielle Diffamierungssoziologie der Justiz und Polizei.« Dgl., Die Arbeit tun die anderen, Klassenkampf und Priesterherrschaft der Intellektuellen, München 1975 , S. 378.

5 Vgl. die Amtliche Begründung des Gesetzes über die einstufige Juristenausbildung in Niedersachsen, Niedersächsischer Landtag. Siebente Wahlperiode, Drucksache Nr. 7/233 I, Hannover 1973, S. $g$ f.

$6 \mathrm{Da}$ ich ein außeruniversitäres Repetitorium nie aufgesucht habe, muß ich mich bei diesem Vergleich auf Berichte von Kommilitonen stützen. Einige Kommilitonen meines Semesters haben den Weg zum Repetitor inzwischen wieder gefunden. 
freundlichsten Falle werden sie als Hilfsargumente akzeptiert und der juristischen

Argumentation äußerlich aufgesetzt. Die Unvermitteltheit der Einführung in die normativen und dogmatisierten Bestände juristischer Argumentation nach der Eingangsphase, der auf den Studenten lastende Leistungs- und Einpassungszwang und die arrogante Ignoranz einzelner, aber exponierter Lehrender haben schließlich, auch bei einem Teil der Studenten, zur fatalen Konsequenz, die reflexionshaltigen Ausgangspunkte des Studiums ganz im Sinne der konservativen reinen Juristen als verzichtbaren Ballast anzusehen, werden doch die dort geweckten Motivationen und Erkenntnisinteressen im Fortgang ohnehin frustriert. In Reaktion darauf und scheinbar der studentischen Kritik am $»$ Dogmatikschock « genügend, diesen aber nur vorverlagernd, haben es die Juristen inz wischen sogar erfolgreich vermocht, mit ihren streng juristisch-dogmatischen Kursen in die Eingangsphase einzubrechen und die sozialwissenschaftlichen Reflexionsgehalte tendenziell auf ein Schattendasein zu reduzieren.

Mit der Reform der Juristenausbildung war - grob zusammengerafft - die Schaffung eines Studienganges intendiert, der effizienter und effektiver, also insgesamt wirtschaftlicher und vor allem stärker auf berufspraktische Erfordernisse einerseits wie auf neue Aufgaben juristischer Praxis andererseits zugeschnitten sein sollte. Der Out-Put, um in der Sprache der Bildungstechnokraten zu verbleiben, sollte in einem Juristen bestehen, dessen Entscheidungen als instrumentell rationaler, von den Entscheidungsprämissen wie der Folgenseite her kalkulierter, ja von den Reformern, die nicht bloße Modernisierung im Sinne hatten, sogar als material rationaler in einem geradezu emphatischen Sinne ${ }^{7}$ vorgestellt wurden. Ausgangspunkt war nämlich die richtige Beobachtung, daß in allen Rechtsgebieten die Abkehr von der positivistischen, rein logisch-hermeneutischen Normanwendung und an deren Stelle die bewußte, aufgeklärte Programmformulierung und -konkretisierung als rechtspolitische Praxis den Normzwecken eher gerecht wird, dies vom entscheidenden Juristen aber größere Fungibilität und Flexibilität erfordert. ${ }^{8}$ Die Effizienz des Studiums glaubten die Reformer durch eine Verkürzung der Studienzeit ${ }^{9}$, die Kontrolle der Effektivität durch ein durchgefeiltes Leistungskontrollsystem (Scheine) gewährleisten zu können. Diese Mittel zur Gewährleistung der Reformziele Effizienz und Effektivität erweisen sich aber als inkompatibel gegenüber dem Ziel des Gewinnes von materialer Entscheidungsrationalität. Die Kürze der Ausbildungszeit wie das Leistungskontrollsystem zwängen die angestrebten Lernprozesse in eine Zeitstruktur hinein, die nurmehr hastige Wissensakkumulation ohne jede Reflexion zuläßt.

Ein trauriges Schnippchen haben die Reformer ihrer mit Emphase intendierten Reform insbesondere dadurch geschlagen, daß sie die Ergebnisse der Leistungskontrollen - zehn einzelne Teilleistungen sind von den Studenten bereits in dem nur dreisemestrigen ersten Hauptstudium im Zivil- und Strafrecht zu erbringen unmittelbar in die Examensendbenotung einfließen lassen wollten. Konsequenterweise mußten sie die Themen und die Bewertung der auch im reformierten Modell unangetasteten und folgenreichen Klausuren institutionell dem Justizministerium bzw. dessen Justizprüfungsamt überantworten und diesem so einen ungeheuren

7 Vgl. statt vieler Reformliteratur der späten 6oer und frühen $70 e r$ Jahre die sympatische und exemplarische Rede des Abgeordneten Pennigsdorf (SPD) im Niedersächsischen Landtag, Stenographische Berichte. Siebente Wahlperiode, 43. Tagungsabschnit,, 89. Sitzung am I8. 12. 1973, Hannover 1974, S. 886s ff.

$8 \mathrm{Vgl}$. die Begründung der Gesetzesvorlage durch den niedersächsichen Justizminister Schäfer (SPD) yor dem Niedersächsischen Landtag, Stenographische Berichte, a. a. O., S. $88_{4} 6 \mathrm{ff}$. (8848).

9 Vgl. die Amtliche Begründung des Gesetzes über die einstufige Juristenausbildung in Niedersachsen, Drucksache Nr. 7/233 I, a. a. O., S. Is. 
Einfluß auf die Prüfungsanforderungen und -gegenstände einräumen. Wer die Prüfungen beherrscht, beherrscht die gesamte Ausbildung. Man kann einer Prüfungsbürokratie nicht vorwerfen, daß sie ihre Möglichkeit offensiv nutzt, über selektive Themenstellung und harte Bewertung von Klausuren auf die Studieninhalte Einfluß zu nehmen. Heute wird das gehandhabte Curriculum - zum Teufel mit der selbstverwalteten Freiheit der Universität! - letztendlich im Justizprüfungsamt gemacht.

Ein Studium, das den Charakter eines Zehnkampfes angenommen hat, muß kritischer Rationalität - vorausgesetzt, rechtsförmige Entscheidungskriterien ließen solcher einen Raum - nicht nur nicht förderlich, sondern schädlich sein. Wenn Prüfungserfordernisse praktisch wirksam werdende Studienmotivationen überdeterminieren, weil von dem Bestehen der Teilleistungsphalanx die Zulassung in die nächste Praktikums- und Studienphase abhängt, muß man zur Erlangung des erforderlichen und umfangreichen kasuistischen Wissens Normenprogramme pauken, ohne daß materiale Prämissen und Entscheidungsfolgen auch nur am Rande eine Relevanz entfalten könnten. In den klausurrelevanten Kursen wird Normanwendung denn auch - die Ausnahme bestätigt die Regel - theorielos, unhistorisch und unter kritikloser Beachtung der herrschenden Lehrmeinung und der höchstrichterlichen Rechtsprechung eingeübt. Jenes distanzierte, normkritische Bewußtsein von der Gewordenheit, Relativität, Zweck- und Interessenbezogenheit des Rechts, das einer zweck- und folgenkalkulierenden Rechtsanwendung vorausgeht, bedarf demgegenüber einer gänzlich anderen Zeitstruktur des Studiums, die eine gleichsam forschende Auslotung der Tiefen und Untiefen des Gegenstandes ermöglicht. In der Reformausbildung, wie sie real existiert, wird durch die dem industriellen Arbeitsprozeß verwandte Zerstückelung der Zeit aber als Sozialisationserfolg sanktioniert, daß Recht den Regeln der herrschenden Norminterpretation entsprechend richtig angewendet, das materiale Entscheidungsergebnis der Beliebigkeit anheimgegeben wird. Die Formalqualifikationen der traditionellen Juristenausbildung geben dabei das Leitbild ab. Der Reformjurist ist am Ende so beschaffen, als hätte er - bei kürzerer Studienzeit - am altehrwürdigen Rechtsfachbereich der Universität Göttingen studiert.

"Die gesunde Wißbegierde wünscht ihren Gegenstand ganz zu fassen, bis in sein Innerstes zu durchdringen und zu zerbeißen «, sagt Friedrich Schlegel in seiner »Lucinde«. Weder im dreisemestrigen ersten Teil des Hauptstudiums am rechtswissenschaftlichen Fachbereich in Hannover noch später kann ein Gegenstand in seinem Innersten durchdrungen werden. Das intellektuelle Zerbeißen der gegenständlichen sozialen Welt hatten schon die Reformer nicht als Aufgabe einer rechtswissenschaftlichen (Aus-)Bildung gesehen. Zwar sieht der Studiengang nach den ersten Praktika für das Studium im siebenten und achten Semester eine sogenannte Vertiefungsphase vor, die Gelegenheit bieten soll, einzelne Problembereiche vor dem Hintergrund der gemachten praktischen Erfahrungen in der Zivil- und Strafrechtsstation genauer und tiefer zu behandeln. Dieses Vertiefungsangebot erscheint mir aber als Zumutung und kaum realisierbar, dienen doch siebentes und achtes Semester im wesentlichen der Vorbereitung auf das Praktikum in der Verwaltung. Hinter den dazu zu erbringenden fünf Teilleistungen und die umfangreichen materiellverwaltungsrechtlichen, verwaltungsverfahrensrechtlichen und verfassungsrechtlichen klausurrelevanten Ausbildungsinhalten tritt das Angebot zur Vertiefung zurück wie eine vergangene Epoche hinter die Gegenwart. Individuelle Studiengestaltung läßt schließlich auch nicht die sogenannte Spezialisierungsphase nach den Verwaltungs- und Anwaltstationen im zehnten und elften Semester zu. Zwar mildert sich hier - nur drei Teilleistungen sind zu erbringen - der Leistungsdruck, und die Studieninhalte sind eher - weil im 
gewählten Studienschwerpunkt - auf die eigenen Interessen zugeschnitten, doch stehen in den nur zwei Semestern bereits in aller Dringlichkeit jene Anforderungen im Vordergrund, die das Staatsexamen erheischt. Forschendes Lernen, wie es in Projektseminaren etwa allzuselten angeregt wird, hat angesichts der aufzuholenden Defizite und Repetitionsnotwendigkeiten keinen Logenplatz.

Die mit der Ausbildungsreform angestrebte Theorie-Praxis-Integration hat der schleichenden, aber nachhaltigen Verkehrung der Reform nicht etwa Einhalt geboten, sondern sie verstärkt. Nachdem nämlich die ersten Reformjuristen nach der dreisemestrigen Hauptphase den Staatsanwaltschaften und Zivilgerichten überwiesen worden waren, meldeten auch die Praktiker ihre Ansprüche an. Statt daß, wie intendiert, von den neuen Juristen ein Hauch von produktiver Verunsicherung und materialer Rechtsrationalität in die juristische Berufspraxis eingegangen wäre, kam ein kalter Wind in den rechtswissenschaftlichen Fachbereich zurück. Nach Auffassung insbesondere der niedersächsischen Staatsanwaltschaften sollte die kasuistische Ausbildung unabweisbar intensiviert, müßte ein deutlicher Rückstand zu den Göttinger Referendaren aufgeholt werden. Der wiederholt vorgetragenen Kritik aus der Berufspraxis, dem neuen Juristen fehlten grundlegende materiellrechtliche Kenntnisse, konnte ein rechtswissenschaftlicher Fachbereich dann auf Dauer auch nicht widerstehen. Obwohl auf der Hand liegt, daß nicht in wenigen Semestern Gleiches zu leisten ist wic in cinem Vollstudium, wurde die reflexionslose Einübung inhaltlich beliebiger, formal aber regelmäßiger Fallentscheidungen quantitativ ausgeweitet und intensiviert. Selbst noch ein spezieller Kurs mit der anspruchsvollen Bezeichnung "Theorie der Praxis «, der dem Praxisintegrationsgebot ${ }^{10}$ entsprechend und in berufspraxiskritischer Weise die zu professioneller Rechtsanwendung notwendigen technischen Fähig- und Fertigkeiten thematisieren sollte, ist in seiner strafrechtlichen Variante in einen materiellrechtlichen Paukurs umgewandelt worden. Die angestrebte Theorie-Praxis-Integration ist so ganz in der Weise, wie sie Stephan Leibfried seinerzeit bereits als Gefahr benannt hatte ${ }^{11}$, zu einer Unterordnung der universitären Ausbildung unter die eingespielten beruflichen Anforderungen vorkommen. Die schwarze Logik der Reform hat sich durchgesetzt.

Daß das Scheitern der intendierten zukunftsweisenden Reform angesichts der Anpassung der Ausbildung an traditionelle berufsständische Juristenbilder und berufsbezogene Fertig- und Fähigkeiten nicht auch zu völliger Dequalifikation und borniertem Reflexionsverzicht auf seiten der Studenten führt, scheint mir dem Ausmaß produktiver Phantasie zuzuschreiben zu sein, das erforderlich ist, um die Fallstricke des Studienganges und seines Leistungskontrollsystemes zu umgehen und zu unterlaufen, aber auch jenem Gefühl eines Mangels, das aus der ständig präsenten Differenz zwischen Reformzielen und schlechter Realität entsteht und überschießende Motivationen zu theoretischer Arbeit auslöst. Überhaupt Zeit zu haben, die in kürzester Zeit zu akkumulierenden Wissensbestände unter Umgehung der rigiden Kursstruktur zu erwerben und die Teilleistungen ohne unangemessenes zeitliches Engagement zu erbringen, ermöglicht den Studenten eine Selbstorganisation in Kleingruppen. Nach meiner Erfahrung sind sie die eigentlichen arbeitenden Körperschaften des Fachbereiches: Der Besuch von Kursangeboten kann aufgeteilt werden, so daß die Rezeption von prüfungsirrelevanten, aber um so interessanteren Studieninhalten möglich wird, Themen von Hausarbeiten und mündlichen Prüfungen

Io Vgl. die Amtl. Begründung ..., Drucksachen Nr. 7/2331, a. a. O., S. 10

I I Stephan Leibfried, Tendenzen der Einphasenausbildung, in: Kritische Justiz Heft 2/1973, S. 182-209 (184); vgl. auch die genaue Darstellung des Hannover-Modells bei Wolfgang Voegeli, Einphasige Juristenausbildung, Zur Pathologie der Reform, Frankfurt/Main, 1979, S. 175 f. 
können abgesprochen und ausgetauscht, Klausuren vorbereitet werden. Schließlich übernehmen sie auch sozialtherapeutische Funktionen, wenn die im Leistungszehnkampf erfahrenen Wunden nicht heilen wollen. Die Fähigkeit, sich mit Phantasie, überlegtem Halbengagement und kluger Risikovermeidung gemeinsam durchwurschteln zu können, scheint mir der eigentliche Sozialisationserfolg der reformierten Ausbildung zu sein, ein Erfolg, der auch in einer traditionellen Ausbildung erreicht werden kann. Zu fürchten ist nur, daß die im Studiengang aufgenötigten Strategien kluger Risikovermeidung nach dem Studium sich allzu oft zu jener Mentalität risikoscheuer Subalternität verfestigen, die Ovid in den Metamorphosen - den mythischen Technologen meinend, doch wie auf den modernen juristischen Sozialtechnologen gemünzt - so charakterisierte: "Hohes vermeidet er bang, des früheren Falles gedenkend."

Stärker aber als die universitäre Ausbildung, von der bisher nur berichtet wurde, prägt den neuen Juristen die praktische Tätigkeit außerhalb der Universität. Wird an der Universität die Relevanz der wie Spielmarken erworbenen Rechtfiguren für Entscheidungsfolgen kaum deutlich, so verwandelt sich hier der Dieb A. und der Hehler B. eines beliebigen Falles in einen wirklichen Menschen, der zum Objekt bloß juristisch-deduktiver Subsumtion nicht mehr taugt. Die graue Sammlung juristischer Spielmarken verliert ihre in der Universität gepflegte Bedeutung angesichts des Reichtums und der Farbigkeit etwa des kriminellen Alltagslebens. In der juristischen Berufspraxis ist denn auch der eigentliche Preis für den Entschluß zu zahlen, sich solcher Ausbildung zu unterwerfen.

Konnte ich im Rahmen des Studiums die politisch-moralische Identität noch dann relativ unangetastet wahren, wenn ich den universitären Erwerb technischinstrumenteller Fertigkeiten von der täglichen Lebenspraxis und der politischtheoretischen Arbeit weitgehend abtrennte, so verwandelt sich die Trennung dieser zunächst konfliktfrei nebeneinander bestehenden Lebenssphären in der juristischen Praxis zu einem manifesten Rollenkonflikt. Anders als das Studium erheischt die praktische Tätigkeit nämlich über die bloße Beherrschung gesetzlicher und dogmatischer Entscheidungskriterien und -techniken hinaus die Einhaltung von sozialen Verhaltensorientierungen und Vermeidungsregel ${ }^{12}$, die der eigentlichen juristischen Entscheidungstätigkeit zwar äußerlich, sozial aber als Katalysatoren des bürokratischen Arbeitsprozesses unerläßlich sind und anders als das Studium persönliche Anpassungsleistungen fordern. Der Kontrollmechanismus am Arbeitsplatz besteht - in dieser Direktheit in der Universität noch undenkbar - in einer unmittelbaren Abhängigkeit und Weisungsgebundenheit zu dem ausbildenden Staatsanwalt, Richter oder Regierungsrat. Die zu beachtenden Vermeidungsregeln und Verhaltensorientierungen kollidieren notwendig mit solchen Symbolen und Attitüden, die den neuen Juristen als Teil einer Studentengeneration ausweisen, der mehrheitlich ein Jeansverbot im Dienst, erstarrte Höflichkeitsformen und weiße Krawatten wie als "Koreapeitsche« bekanntgewordene Kurzhaartracht im Gerichtssaal nichts mehr sagen. Solche Anpassungsanforderungen in äußeren Symbolen und Attitüden scheinen banal zu sein. In der konkreten Situation eines Praktikums jedoch bilden sie gleichsam das Äußere einer frontier, die sich in einem selber austrägt. Symbole der Nichtanpassung und Verweigerung können hier erforderlich sein, um sich seiner selbst zu vergewissern, symbolisieren die erwarteten katalysatorischen Verhaltensweisen doch gerade jene Selbstverpflichtung zu loyaler juristischer Sozialkontrolle,

12 Diesen Begriff hat Claus Offe, Leistungsprinzip und industrielle Arbeit, Frankfurt/Main, 1970, S. 40 zur Charakterisierung von regulativen, extrafunktionalen Normen im industriellen Produktionsprozeß benutzt. 
die zu erbringen dem eigenen besseren Wissen widerspricht, die man um des Studienabschlusses willen aber mindestens für kurze Zeit erbringen muß. Am krassesten und damit am eindrucksvollsten hat das Problem des Rollenkonfliktes auf der symbolischen Ebene ein Kommilitone in einem Erfahrungsbericht in der studentischen Fachbereichszeitung beschrieben. Der Artikel, unterzeichnet von dem Stadtindianer Chironimo, trägt die Uberschrift: "Tomahawk und Staatsanwalt «.

„Der weiße Mann wollte nicht nur, daß wir machen, was er uns vormacht, er wollte, daß wir völlig wie er werden. Zuerst sperrten sie uns in riesige Kästen aus Glas und Stein, wo kein Wind unser Haar zausen und keine Sonne unsere Haut bräunen konnte. Dann mußten wir in Bergen von Papier wühlen, damit der Staub sich auf unseren Gesichtern niederlege und wir genauso grau und tot aussehen wie er. Wir sollten uns weiße Stricke um den Hals schnüren, die uns röcheln machen, und ein Tuch von der Farbe des Todes um uns hängen, damit unsere bunten Stammeskleider nicht mehr zu sehen waren. Unsere blumenreiche Sprache wurde verboten, wir sollten die Sprache der Bleichgesichier sprechen, damit keiner unserer geschundenen Brüder und Schwestern uns mehr versteht. [...]

Aber wir haben alle diese Martern ertragen.

Doch dann wollten sie den Skalp eines unserer Brüder. Sie wollten, daß er sich blau scheren ließe wie sie, sonst wollten sie ihn noch mehr strafen als alle anderen Brüder und Schwestern.

Doch kein Indianer irgendeines Stammes gibt seinen Skalp freiwillig.

Die Brüder und Schwestern Jießen ihren Bruder nicht allein. Sie waren bereit ihre Tomahawks zu benutzen und teilten dieses den Bleichgesichtern mit.

Da war das Wutgeheul groß. Sie fingen an zu lügen und wanden sich. Sie sagten, der Bruder solle seinen Skalp gar nicht hergeben. Er werde nur bestraft, weil er seine Arbeit schlecht gemacht habe (oh, gespaltene Zunge: Sie meinen ihre Arbeit). Aber niemand glaubte den Weißen und sie quälten den Bruder weiterhin. Aber der Bruder blieb standhaft und behielt seinen Skalp. Dafür durfte er dann ihr schmutziges Geschäft nicht üben. Er sollte froh darüber sein. ${ }^{13}$

Zu Unrecht wird der Zusammenprall zweier (auch) politischer Kulturen aber in einem Praktikum von vielen alternativ-linken Kommilitonen auf die symbolische Ebene begrenzt. Wichtiger und tiefgreifender ist nach meiner Erfahrung der politisch-moralische Preis, der in der eigentlichen juristischen Entscheidungstätigkeit über Anklageerhebung, Strafmaß oder zivilgerichtliche Urteilstenorierung selber zu entrichten ist. Hier nämlich entsteht das Gefühl, sich die Hände beschmutzt und moralische Schuld auf sich genommen zu haben, aus der realen Verantwortung für getroffene Entscheidungen. Daß dieses Gefühl nicht Produkt eines übersteigerten moralischen Rigorismus sein muß und nicht erst dann entsteht, wenn der politische Charakter justizieller Entscheidungen ganz explizit ist, mag folgende Episode beleuchten.

Während meines Pflichtpraktikums bei der Staatsanwaltschaft waren einige Auftritte und Plädoyers als Staatsanwalt ohne Beaufsichtigung obligatorisch. In einem Fall war ein alter Mann angeklagt, der sich, vom Kriege entwurzelt und von der nachfaschistischen Leistungs- und Konsumgesellschaft ausgespien, wiederholt an privatem und öffentlichem Eigentum vergriffen hatte. Das beharrliche Schwarzfahren mit der Bundesbahn zur Steigerung seiner Mobilität und zahlreiche Kleindiebstähle und Betrügereien zum Lebensunterhalt hatten seinen Zentralregisterauszug bald mit Leben erfüllt. Er war ein harmloser, lieber Kerl, mit der Subkultur der Schlafhäuser und Vollzugsanstalten gut vertraut; er stand mit treuen schuldbewußten Augen vor mir, dem jungen neuen Staatsanwalt. Der Anklagesatz warf ihm vor, eine PrinzHeinrich-Mütze in einem Warenhaus gestohlen zu haben. Auf die Frage des Richters nach dem Warum antwortete er bereitwillig, er habe auch einmal so eine Mütze haben wollen, wie sie der Bundeskanzler Schmidt immer trage.

I3 Chironimo, Tomahawk und Staatsanwalt, in: Paragraphenkotzer, Zeitung an der Fak. VI, Nr. 8, Hannover 1978. 
Ein Kleindiebstahl im Werte von etwa dreißig Mark wird in jenem Landgerichtsbezirk in der Regel mit einer Geldstrafe von zwanzig bis dreißig Tagessätzen bestraft. Für mich lag auf der Hand, daß die Bestrafung des Mannes aus spezialpräventiver Sicht ohne jeden Sinn war. Nichts und niemand würde ihn auf den rechten Weg zurückbringen, solange eine Sanierung seiner finanziellen Situation diesen Weg nicht absicherte. Der alte Mann war aber Wiederholungstäter. Der allgemeine Teil des Strafgesetzbuches sieht unerbittlich die Freiheitsstrafe von mindestens einem halben Jahr vor. Wie sich in einer solchen Situation entscheiden, ohne an einem sinnlosen Freiheitsentzug, dem in dieser Republik bislang einschneidensten Eingriff in eine Lebensgeschichte, mitschuldig zu werden? Die Delegation der Entscheidung auf einen Sachverständigen, der gern gewählte Ausweg der Psychatrisierung kam hier nicht in Betracht, war der Mann doch unzweifelhaft völlig »normal«. Ich habe mich als Jurist verhalten und die halbjährige Freiheitsstrafe beantragt. Da erregte sich das Opfer meiner Funktionstreue, sein milieubezogenes Unrechtsbewußtsein sagte ihm, daß hier moralisches Unrecht geschah. Sein Schlußwort war von verblüffender Plausibilität: Bestraft werden müsse er schon, aber wenn er wegen einer Kanzlermütze ein halbes Jahr sitzen müsse, dann werde er selbst von seinen besten Freunden ausgelacht. Wäre ich nicht zur Rollenkonsistenz verpflichtet gewesen, hätte ich ihm zugestimmt, stand mir sein Rechtsbewußtsein doch näher als alle Lehrbuchmeinung $\mathrm{zu} \ 48$ des Strafgesetzbuches. So aber hörte ich mir das meinem Antrag entsprechende Urteil an, sah, wie der Alte abgeführt wurde und fühlte mich schlecht.

Die beschriebene Situation beleuchtet nach meiner Erfahrung nicht ein nur der juristischen Tätigkeit in der »Strafrechtspflege« zuzuordnendes Sonderproblem. Ich habe in der Zivilgerichtsbarkeit, in der Verwaltung und - in anderer Konstellation auch in der Anwaltstätigkeit immer wieder solch dilemmatische Situationen durchlebr, ob sie nun kündigungsschutz-, datenschutz- bzw. immissionsschutzrechtliche Probleme betrafen oder die Weisung von Vorgesetzten statt gesetzlicher Bestimmungen ein opportunes Verhalten einforderte. Sicherlich geht die Tätigkeit von Juristen in solchen Konfliktlagen, die mit der Antinomie von Gesinnungs- und Verantwortungsethik ${ }^{14}$ nur unzureichend zu fassen sind, nicht einfach auf. Es mögen sogar Ausnahmesituationen sein, die aber geeignet sind, das Grunddilemma der juristischen Entscheidungstätigkeit aufzudecken. "Wo der demokratische Anspruch einmal erhoben ist, «- so hat Hans-Erich Troje dieses Dilemma gekennzeichnet - "kann man nicht länger mit Menschen wie mit Nichtmenschen oder Menschen zweiter Klasse umgehen. " $^{15}$ Der neue Jurist muß aber wie jeder Ju rist lernen, mit Menschen "wie mit Sachen ${ }^{16}{ }^{6}$ umzugehen, erforderte Anderes doch mehr als eine Reform der Juristenausbildung. Die Form, in der sich Juristen als Rechtsanwender auf Menschen als bloß gehorsame Regelungsobjekte beziehen, genügt nicht den Ansprüchen demokratischer und egalitärer gesellschaftlicher Verkehrsformen. Weil Rechtsanwendung mehr ist als nur Vollzugshandlung von parlamentarisch legitimierten Rechtssetzungen, greifen angesichts dilemmatischer Entscheidungssituationen individuelle Entlastungsstragegien zu kurz, die die Verantwortung für die Entscheidungsfolgen auf den Gesetzgeber und seine legislativen Entscheidungen abwälzen. Ging es bei der oben beschriebenen symbolischen Abwehr von Verhaltenszumutungen darum, den Schein der Kumpanei mit denen, die herrschen, nicht symbolisch sichtbar werden zu lassen, so wird mit der Form juristischen Entscheidens, wie sie den politisch-moralisch

I4 So aber Helmut Schelsky, Die Arbeit tun die anderen, a. a. O., S. 380 . Solche Konfliktlagen spiegeln die gesellschaftspolitische Blockadesituation wider, der die Republik in ihrer zweiten Restaurationsperiode unterliegt.

is Hans-Erich Troje, Juristenausbildung heute, Neuwied/Darmstadt, S. $108 \mathrm{f}$

16 Ders., ebd., S. 108. 
unvertretbaren Entscheidungen zugrundeliegt, Herrschaft selber ausgeübt. Herrschaft hat aber, mag sie auch in Institutionen auf Dauer gestellt und System geworden sein, immer noch das personale Moment zur Bedingung. Dieses - sicher austauschbare - Moment beinhaltet zugleich auch immer mindestens einen Rest von Verantwortung dessen, der die Entscheidung trifft. ${ }^{17}$

Der geschilderte Rollenkonflikt, dessen Dilemma sich in der eigenen Person austrägt und im Grunde von jedem Juristen bei Entscheidungen über Lebensgeschichten und Schicksale anderer verarbeitet werden muß, hat sich mir als nachhaltigster Sozialisationsmechanismus der juristischen Ausbildung eingeprägt. Das Dilemma der Verantwortung für Unverantwortliches kann mit Zynismen überlagert werden, wie ich sie bei Staatsanwälten häufiger angetroffen habe, es kann sogar, wie ich es auch beobachten mußte, zerstörend auf die eigene wie immer auch brüchige und widersprüchliche Identität wirken. Kaum gerecht werden wird man dem Konflikt zwischen juristischer Berufsrolle und politisch-moralischer Identität aber auch mit dem forsch-pragmatischen Pseudorealismus von well-adjusted people, die immer in Vorausanpassung schon wissen, was von sich selber sie aufgeben müssen, um danach erleichtert festzustellen, daß alles so schlimm nicht war. Solches Vademecum schützt zwar vor offenen Konflikten, Selbstbeschwichtigung beseitigt aber nicht jeden inneren Zwiespalt. Produktiv wird man die gemachte Erfahrung wohl nur dann machen können, wenn man sich ihr stellt, den Zwiespalt aushält, den gesellschaftlichen Blockade- und Krankheitszustand, der hier gelebt werden muß, als solchen diagnostiziert, ohne sich von aller Verantwortung mit Verweis auf Gesetz und System zu entlasten.

Immerhin steckt auch eine Chance in der so verarbeiteten Erfahrung mit juristischer Praxis. Die Erfahrung des Zwiespaltes verhindert eine Anpassung, die schon der Studiengang mit seinem Gratifikationssystem dadurch erkauft, daß er die fortschreitende Verstrickung an ein Wachstum von Entgelt - in den letzten beiden Studienjahren werden Dienstbezüge gezahlt - und Macht koppelt. Die aufkommende Lust an der Macht wird zur Last. Die Erfahrung des täglichen Umgangs mit der Macht in staatlichen Institutionen verändert zugleich die Perspektive bloßer Ohnmacht. Sie hilft nämlich, jene Ängstlichkeit und Simplizität zu vermeiden, mit der einige, aber für einen Teil der politischen Linken exemplarische Kommilitonen den Staat mit einem in sich geschlossenen, opaken und böswilligen Behemoth kurzerhand identifizieren und den eigenen politischen Ort abseits der »verstaateten « Gesellschaft in einer ständig bedrohten und marginalisierten Peripherie bestimmen. Solche Selbstausgrenzung aus dem gesel]schaftlichen Erfahrungszusammenhang, die Lagermentalität der Aussteigerideologie, hat sich mit dem ohnmächtigen und peripheren Dasein schon abgefunden. Die Erfahrung mit der Brüchigkeit und Widersprüchlichkeit des politischen Herrschaftssystems, der Koordinationsprobleme und intellektuellen Anspruchslosigkeit seiner repressiven Apparate, wo nur der äußere Anschein effizienter Aufgabenerfüllung herrscht, selbst soweit sie direkt kapitalistische Interessen betreffen, bewältigt die Angst, nurmehr Opfer anonymer Macht zu sein, - und ist damit Ermutigung.

Josef Brink

I7 Obwohl sich der bürgerliche Jurist durch Gewaltenteilung und Beamtenrecht aus dem unmittelbaren Abhängigkeitsverhältnis zum absolutistischen Herrn teilweise emanzipiert hat, ist doch die Form seiner Beziehung zum Regelungsobjekt Mensch der absolutistischen Tradition verhaftet. Sozialtechnologie Luhmannscher Prägung mag das Subjekt, das entscheidet, gänzlich in systemfunktionale, rollenmäßig ausdifferenzierte Objektivität zerstückeln wollen, in der Entscheidungssituation muß der entscheidende Jurist als ganze Person verantwortlich zeichnen, ganz so, wie er den Dieb als willentlich und wissentlich Verantwortlichen verurteilt. 


\section{Ambivalenz der Ausbildungsreform - Auseinandersetzung mit einem Erfahrungsbericht}

In der mittlerweile mehr als ein Jahrzehnt währenden Diskussion um die Reform der Juristenausbildung ${ }^{\mathrm{I}}$ sind auch in den durch die Verlängerung der Experimentierpha$\mathrm{se}^{2}$ provozierten Beiträgen der jüngsten Zeit praxisbezogene Erfahrungsberichte betroffener Studenten so gut wie nie publiziert worden. Ausbildung, so scheint es, bleibt auch hier ganz Domäne der Profession, die von ihren Veränderungen am wenigsten betroffen ist.

Allein deshalb muß es begrüßt werden, wenn über Artikel in hochschul- oder auch nur fakultätsinternen Blättern ${ }^{3}$ hinaus studentische Erfahrungen mit der neuen einstufigen Juristenausbildung einem größeren Lesepublikum unterbreitet werden - nicht zuletzt deswegen, weil die häufig an der Leine abstrakter Prinzipienstreiterei verlaufende Debatte der Anreicherung mit etwas konkreterem Erfahrungsstoff durchaus bedarf.

I.

I. Das Hauptmanko des Berichts von Brink (B.) sehen wir darin, daß er an keiner Stelle eindeutig expliziert, an welchen Maßstäben eigentlich die beschriebene Ausbildung im Hannover-Modell (HM) einer Prüfung unterzogen wird. Unklar bleibt, ob B. die schlechte Realität der Ausbildung an den - prinzipiell für gut befundenen - Ansprüchen und Konzepten des Modells, die sich leider real nicht haben durchsetzen können, kritisiert oder ob seinem Verdikt bereits die Konzeption der Ausbildung auf Programmebene verfällt. Weil B. nicht deutlich genug herausstellt, ob er die unzureichende Einlösung der richtigen Postulate oder die Postulate als falsche Versprechen kritisiert, gerät ihm seine Bestandsaufnahme unter der Hand zu einer Summation negativer Erfahrungen und punktueller Unlusterlebnisse, ohne daß deren Negativqualität an konsistenten Kriterien aufgewiesen würde. ${ }^{4}$

Das Hauptstudium I ist für B. im extremsten Maße Stätte der Dressur, der unreflektierten Vermittlung von Dogmatik, die unangegriffen und unhinterfragt gepaukt wird. Die folgenden Praktika schildert B. als Reihe von Kaltwassersprüngen,

I Aus der Anfangsphase, in der die neuen Konzepte offensiv vorgetragen wurden, sind zu nennen: Loccumer Arbeitskreis (Hrsg.), Neue Juristenausbildung, Neuwied/Berlin 1970; Der neue Jurist, Ausbildungsreform in Bremen als Planungs- und Lernprozeß, Darmstadr/Neuwied 1973; starke Bedenken schon zu Beginn der Modellerprobung bei Stephan Leibfried, Tendenzen der Einphasenausbildung, KJ 2/1973, S. 182-209; einen guten Gesamtüberblick gibt Wolfgang Voegeli, Einphasige Juristenausbildung. Zur Parhologie der Reform, Ffm. 1979; aus jüngster Zeit: Hans- Georg Salge, Reform der Juristenausbildung, Anspruch und Wirklichkeit, DRiZ 1980, S. 4 I-49; Dieter Hart, Vereinheitlichung

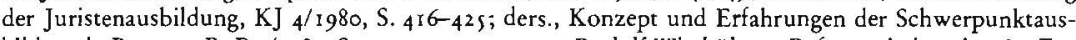
bildung in Bremen, RuP $3 / 1980$, S. 144-149; neuestens: Rudolf Wiethölter, *Reformatio in peius $*$ ? - Zur Geschichte der Ausbildungsreform, KJ 1/1981, S. I-17.

2 Die Änderung erfolgte durch das Zweite Gesetz zur Anderung des Deutschen Richtergesetzes; vgl. die kurze Darstellung in ZRP 1980 , S. 2 I 1 .

3 Am Fachbereich für Rechtswissenschaften in Hannover z. B.: "Der Paragraphenkotzer« mit bislang ${ }_{3}$ Ausgaben seit Dezember 1976 .

$4 \mathrm{Zwar}$ sollen die Schwierigkeiten der Erbringung einer in diesem Sinn konstruierten (nicht notwendigerweise: konstruktiven!) Kritik nicht unterschätzt werden: Uber auf Plausibilitätsniveau stehenden Einschätzungen wird man beim derzeitigen Stand der Dinge nicht hinausgelangen können. B. aber beläßt es insgesamt bei der Artikulation eines allgemeinen Unbehagens an der Juristenausbildung schlechthin, 
die unvorbereitet und ohne Assistenz der Fakultät erlitten werden müssen: Wie in der

Uni dem Paukbetrieb, ist man nun der Praxis hilflos ausgeliefert.

Welche Schlüsse, so fragt man sich, sind aus diesen Einschätzungen zu ziehen? Aus der ersten wohl ein Zurückdrängen der Dogmatik, weniger pragmatisch orientiertes Handwerkszeug, stattdessen mehr kritische Reflexion, Hinterfragung anstelle von kritischer Meinung. Dies würde aber die Gefahr eines noch geringeren Maßes an praktischer juristischer Kompetenz begründen und die Schwierigkeiten bei der Praktika-Bewältigung eher noch erhöhen. Steuerte man dem aber durch eine bessere und intensivere, und d. h. doch zumindestens auch: dogmatische Vorbereitung in der Universität entgegen, steigerte man die Dogmatik-Dressur bis zur unerträglichen Perfektion.

Von nur vagem Aussagegehalt bleibt auch die Bezugnahme auf die vom HM ",angestrebten Lernprozesse“ oder die „Reformintentionen“. Denn es wird nicht klar, ob B. die tatsächliche, vollständige Verwirklichung der Reformpostulate als begrüßenswerte Innovation gutgeheißen hätte oder ob er den Jetztzustand als die eigentliche, wahre Realisierung der (immer schon allein auf Technokratenproduktion ausgerichteten) „Reform“-Idee rezipiert. Die letzte These wäre nicht ohne Plausibilität: In der Tat drängt sich die Frage auf, ob der Typus eines dogmatisch hinreichend geschulten, technokratisch halbwegs versierten Jungjuristen als Modellprodukt eher als Verkehrung oder lediglich als Untererfüllung der Reformintentionen zu werten wäre. Hat sich, m. a. W., nicht vielleicht das wahre Ziel des HM Gestalt verschafft in jenem stromlinienförmigen, dynamischen, geistig tänzelnden Juristentyp, der seine beliebige Verwendbarkeit noch dadurch um einiges steigert, daß er flink und versiert mit sozialwissenschaftlich aufgeputzten Versatzstückargumentationen hantieren kann: ohne eigene Meinung, aber bereit, jede zu vertreten?

B. läßt uns über seine Ansicht zum realen und intendierten output des HM dauerhaft im unklaren.

2. Nun kann und darf man von einem Erfahrungsbericht kein ausgefeiltes Curriculum erwarten. Bei B. jedoch unterbleibt nicht nur die Entwicklung einer konsistenten Argumentationsbasis - er bedient sich darüberhinaus einer streckenweise kaum noch gezügelten Erlebnismetaphorik, die nicht nur wegen ihrer inhaltlichen Beliebigkeit gefährlich, sondern zum systematischen Aufweis von Defiziten oder auch der Untauglichkeit des Modells in toto untauglich ist. Dem weltfremden abstraktiven Zusammenhang der Normensysteme werden scheinbar konkrete Formen der Lebendigkeit entgegengestellt: entweder als Wissenschaften selbst ("Wirklichkeitswissenschaften «) oder als studentische Interaktion (Kleingruppen, Diskussionen in der Eingangsphase). Der freie Flug der Sozialwissenschaften fungiert bei B. als unerreichbares Leitbild der zurechtgestutzten flügellahmen Jurisprudenz.

Dem Recht wohnt per definitionem nichts Innovatives, Revolutionär-Befreiendes innes, zur individuellen Selbstfindung und/oder -verwirklichung stellt es das denkbar

das sich eher zufällig am HM festmacht. Den Berichten liegen im übrigen nicht völlig identische Realitätserfahrungen zugrunde: In der - von den Verfassern miterlebten - Startphase « des Modells waren weder die Strukturen der Ausbildung irgendwie festgelegt noch der Reformwille der Mehrheit der Dozentenschaft entscheidend geschwächt; die Neustrukturierung der Kernmaterien des Rechts etwa stand noch auf der Tagesordnung. Die sukzessive erfolgten Beschneidungen der Reformintentionen sind von den Verfassern eher als Verlaufsprozesse, von B. wohl stärker als ein Stück bereits fertiger und vorgefundener Realität rezipiert worden.

s "Recht ist das Urphänomen irrationaler Rationalität. In ihm wird das formale Åquivalenzprinzip zur Norm, alle schlägt es über denselben Leisten... Schon der bloßen Form nach, vor Klasseninhalt und Klassenjustiz, drückt es Herrschaft, die klaffende Differenz der Einzelinteressen vor dem Ganzen aus, in dem sie abstrakt sich zusammenfassen. Das System selbstgemachter Begriffe, das die ausgereifte 
ungeeignetste Mittel dar. Wenn B. die Vermittlung des juristischen Wissens als Generalisierungsprozeß beschreibt, in dem alle sich nicht dem System fügenden Inhalte eingeebnet werden, so ist dies weder ein sonderlich bemerkenswerter noch ein für das HM typischer Vorgang ${ }^{6}$. Damit ist nicht die Behauptung einer völligen Indifferenz des Inhalts des materiellen Rechts impliziert: Wie in Teil II noch auszuführen sein wird, sehen wir die Wahrnehmung von Handlungs- und Dispositionsspielräumen in der Praxis nicht als von vornherein chancenlos an. Gerade dies setzt aber die Handhabung des Rechts als Recht voraus: Indem man sich so notwendigerweise im Medium des Rechts bewegen muß, unterwirft man sich zwangsläufig seinem strukturellen Konservatismus. Wer diese Fundamentalbestimmung übersieht und die erstaunt zur Kenntnis genommene Unfreiheit der Jurisprudenz entsetzt als Defizit einer juristischen Ausbildung ausgeben will, ist entweder naiv oder erliegt einer Verwechslung von Ursache und Wirkung.

3. Dennoch ist gar nicht zu leugnen, daß mit der Reform der Juristenausbildung auch anderen Sozialisationsmustern als den herkömmlichen zum Durchbruch verholfen werden sollte - aber nicht unter Abschaffung jener »natürlichen « Deformationen, die bei professioneller Aneignung des Rechts unvermeidbar sind. Die Reform wollte nicht die Jurisprudenz einschließlich aller ihr innewohnenden Sozialisationsmuster abschaffen, sondern funktionstüchtige Juristen produzieren, die für bestimmte (Reform-)Inhalte stehen und einige alte Zöpfe abschneiden sollten. Von der zutreffenden Uberlegung ausgehend, daß ein feindlicher Block der Justiz und Teile der Exekutivbürokratie die Durchsetzung gesamtgesellschaftlicher Reformen unmöglich machen würde, wurde die Ausbildungsreform als ein unverzichtbares Element der Politik der inneren Reformen begriffen: Den sozialen und demokratischen Juristen sah man als conditio sine qua non des sich schrittweise erneuernden und verbessernden Gesellschaftssystems an. Weil Offenheit für neue Entwicklungen und Aufgeschlossenheit für Veränderungen unabdingbare Voraussetzungen des Gelingens dieses Konzeptes waren, sollten Sozialisationserfolge durchaus eintreten: im Sinne des Aufbrechens verkrusteter, dogmatisch-doktrinärer Autoritäts- und Obrigkeitsgläubigkeit, einer weltfremden Prinzipienreiterei ${ }^{7}$ und eines professionellen Konservatismus ${ }^{8}$.

Doch all diese avisierten Reformziele nehmen sich angesichts einer Kritik, die außer einem Lob für die sozialwissenschaftliche Eingangsphase nichts Gutes über das HM zu berichten weiß, geradezu beklemmend nichtig und unwesentlich aus. Das mag nicht anders möglich sein, wenn man die Nähe zum wahren, echten Leben zum Maß aller Dinge nimmt: In B.'s Perspektive sind die Wirklichkeitswissenschaften tatsächlich in der Vorhand, erscheint die juristische Bewältigung und Behandlung der Wirklichkeit als abstraktives, aber feingesponnenes Netz, das sich über den »Block wirklichen Lebens" (Negt) legt.

Jurisprudenz vor den Lebensprozeß der Gesellschaft schiebt, entscheidet sich durch Subsumtion alles Einzelnen unter die Kategorie vorweg für die Ordnung, der das klassifikatorische System nachgeahmt ist. “ (Theodor W. Adorno, Negative Dialektik, Ffm. 1975, S. 304/305)

6 Rudolf Wiethölter, Rechtswissenschaft, Ffm. 1968, bezeichnet das Recht als "Kulturmacht « (S. 28) autoritärer und autoritativer Art: »Recht ist Autorität. Autorität vermittelt Befehl und Gehorsam, setzt Macht frei.“ (S. 32).

7 Wiethölter (Fn. 6) hat diesen Aspekt der Reformbewegung als einer ihrer Mitautoren eindringlich geschildert: Die Rechtswelt lebt »isoliert in einer nachemanzipatorischen Gesellschaft ein voremanzipatorisches Dasein*, ihr ist »der Durchgang von einem idealistisch-philosophisch-bürgerlich-liberalen Zeitalter zu einem pluralistisch-politisch-demokratisch-sozialen Zeitalter nicht bewußt" geworden (S. 35); «nser Recht ist bewußtlos, und niemand hilft ihm zu Bewußtsein zurück ...«(S. 27); Recht hat weder den Anschluß an die Wissenschaften der Gegenwart noch an diese Gegenwart selbst gefunden * (S. 4 I ).

8 Wolfgang Kaupen, Die Hüter von Recht und Ordnung, Neuwied/Berlin 1969 (2. Auflage 1971). 
Einem Abgrund an stupider Juristendressur im Hauptstudium I wird die vergnüg-

liche Idylle einer kommunikativen, lust- und lernvollen Eingangsphase, die sich ihre wissensträchtige Unschuld einem bornierten Wissenschaftsbetrieb gegenüber noch hat erhalten können, entgegengestellt.

Die von den Autoren noch erlebte Konzentration der Sozialwissenschaften in der Eingangsphase bringt das Problem mit sich, daß deren Bedeutung in bezug auf die Rechtswissenschaft wegen des geringen Kenntnisstandes über dies nur schwer nachvollziehbar ist. Deshalb ergeht es einem zuweilen wie dem berühmten Blinden, der über die Farbe spricht: Ohne positive Rechtskenntnis bleibt entweder nur die Beschränkung darauf, die Eigenlogik der Sozialwissenschaften ohne nachprüfbaren Bezug zum Recht zu erfassen oder sich den inhaltlichen Urteilen der Sozialwissenschaftler über das Recht anzuschließen. Welche Verwirrung dies stiften kann, hat plastisch der Student K. O. beschrieben:

nIn den ersten beiden Semestern wurde uns das bestehende Recht als etwas zerbröckelndes, an allen Ecken und Enden aus den Fugen krachendes Gebilde beschrieben, das zwar einer frühkapitalistischen Gesellschaft adäquat sei, aber in die heutige Zeit nicht mehr passe. Die Sozialwissenschaften sollten uns das Instrumentarium in die Hand geben, die Bruchstellen des Rechtssystems aufzuspüren und ein neues, soziales Recht zu schaffen. Es schien also völlig klar: Ohne Sozialwissenschaften geht es mit dem Recht nicht mehr weiter. Mit dieser beinahe sträflichen Naivität kamen wir in das dritte Semester. Hier wurde das von uns totgeglaubte Recht aber erstaunlich lebendig, wir wurden total überfahren, waren hilflos gegenüber dem, was uns da aufgetischt wurde: Das Zivilrecht und das Strafrecht erwiesen sich als geschlossene, scheinbar unangreifbare Systeme. «9

Ungeachtet dieser - durch zwischenzeitlich erfolgte Curriculumrevisionen beseitigten - Gefahr einer informationslosen Vorverurteilung der Rechtswissenschaft durch die Gesellschaftswissenschaften: Bei den Wirklichkeitswissenschaften handelt es sich ebenfalls um abstrakte wissenschaftliche Disziplinen, die die konkrete gesellschaftliche Empirie in ihre allgemeinen Raster und Schemata pressen.

Für eine Verklärung der Sozialwissenschaften zu einer unterd rückten, aber potentiell befreienden Wunderwaffe zur Uberwindung der herkömmlichen Jurisprudenz besteht jedenfalls kein Anlaß.

4. B.’s Charakterisierung der Praktika schließlich ist durch die Überhöhung eines an sich banalen Rollenkonfliktes gekennzeichnet, der bei nüchterner Betrachtung sicher zu prognostizieren und daher ohne Überraschungseffekt war. Oder hätte B. etwa angenommen, daß angesichts des HM die Praxis nur auf die Einnahme ihrer Bastionen durch eine Linkskultur wartet?

Darüberhinaus offenbart die Unschulds-Figur ein »Rühr-mich-nicht-an«-Syndrom, das den Kontakt mit der Praxis nur als eine Art Infektionserlebnis begreifen kann. Dem ist entgegenzuhalten, daß die relativ früh einsetzenden Praktika die produktiv zu nützende Chance des stückweisen Illusionsverlustes bei gleichzeitiger Stabilisierung eines alternativen Handlungsspektrums in sich bergen, die es zumindestens in dieser Form in der traditionellen Ausbildung nicht gibt.

Alles in allem kritisiert B. nicht speziell das HM, sondern (juristische) Ausbildung schlechthin. Sein Bericht ist eine vehemente Polemik gegen die Einebnung und Abstraktifizierung der natürlichen Lebens- und Verkehrsformen durch unstoffliche Systeme; er polemisiert gegen das Recht, seine Anwendung und die Ausbildung hierzu im Namen einer anderen, besseren Welt, die des Rechts als Form abstrakter Synthesis nicht mehr bedarf. In dieser Perspektive kann nichts mehr Bestand haben, 
was auf der Basis des $Z$ wangs beruht; es wird per se zum kritikwürdigen Unterfangen der Etablierung gewaltförmiger Zusammenhänge. Konsequenterweise kann aus dieser Perspektive nicht mehr nach einer besseren oder schlechteren Form der Juristenausbildung gefragt, sondern nur noch der Zeitpunkt ihrer Abschaffung - wie der von Recht überhaupt - diskutiert werden.

II.

r. Im Gegensatz zu B. gehen wir davon aus, daß für Ausbildung immer noch das Floh-Dressur-Beispiel ${ }^{10}$ die treffendste Metapher ist, und zwar für ein- wie zweiphasige Juristenausbildung: $\mathrm{Zu}$ diskutieren bleibt allein über die Höhe der möglichen Sprünge. Differenzen der Modelle sollten unseres Erachtens nicht kriterienlos plangeschliffen werden.

Das HM konzipiert ein Reformstudium, in dem sich die Widersprüchlichkeiten des allgemeinen Formelkompromisses des $₫ \varsigma b$ DRiG (H.-A. Hesse: »Konsens auf höchster Abstraktionsebene «) bis in die letzten Fasern der Modellkonzeption unausgetragen verlängert haben. Schon auf der Modellebene existiert hier eine Ausbildung ohne konsequente Prioritätensetzung. Weder wird - wie in Bremen - der radikale Bruch mit der traditionellen Ausbildung gewagt noch - wie etwa in Augsburg - unter Absage jeglicher inhaltlicher Reform schlichte Effizienzsteigerung betrieben.

Unsere Vermutung ist, daß das HM andere als straditionelle $<$ Juristen produziert, auch wenn Strukturunterschiede hinsichtlich der ,Sozialisationsergebnisse nur unter Verzicht auf eine seriöse Analyse benannt werden könnten. Die Annahme stützt sich auf eine von der Beschreibung B.'s abweichende Wahrnehmung und Interpretation von Studienerfahrungen, wie an einigen Beispielen erläutert werden soll.

2. Nach B.'s Darstellung der Sozialisationsprozesse und -wirkungen der Justizpraktika unterscheidet sich der Modelljurist vom traditionellen allenfalls durch ein höheres Maß an dogmatischer Inkompetenz und technokratischer Willfährigkeit. Für uns dagegen sind die beschriebenen Anpassungsleistungen nur Ausdruck dafür, daß Theorie nicht Praxis ist, daß in der Institution Universität andere Verhaltensmuster, Wertorientierungen gelten und auch anderes äußeres Auftreten verlangt wird. Jeder Referendar, jeder Berufsanfänger hat für sich die Frage zu beantworten, wieweit die Adaption berufspraktischer Verhaltensmuster getrieben werden soll.

Die Mikroebene der "Disziplinierung der gelehrigen Körper " (Foucault) und der Äußerlichkeiten verweist B. zwar zu Recht auf die symbolische Ebene und fragt nach den Inhalten der Arbeit. Nur darf er auch dort nicht auf der bloß negierenden Haltung in der Hoffnung verharren, seine saubere universitäre politische Identität vor dem schmutzigen Geschäft der Praxis retten zu können. An dieser Schnittstelle zwischen Theorie und Praxis prallen nicht nur die sozialen Systeme Praxis und Universität aufeinander; gefragt ist auch die Fähigkeit, in der eigenen Person die Integration der unterschiedlichen Rollenanforderungen leisten zu können. Weniger

Io "Die Juristenausbildung gleicht, wie mir einmal Professor Redslob, der erste Rektor der FU und keineswegs ein fortschrittlicher, sagte, der Dressur von Zirkusflöhen. Die werden nämlich, nachdem man sie gefangen hat, in eine Zigarrenkiste gesperrt, auf die man eine Glasscheibe legt. Wenn die Flöhe versuchen, aus der Kiste zu hüpfen, stoßen sie sich an der Scheibe. Nach einiger Zeit lernen sie, wie hoch sie springen können, ohne sich zu stoßen. Wenn man jetzt die Scheibe abnimmt, haben sie sich abgewöhnt, aus der Kiste zu springen. Dieser Vorgang wird in immer niedrigeren Kisten wiederholt, bis die Flöhe so wenig Platz haben, daß sie überhaupt nicht mehr springen können. Wenn sie dann gelernt haben, sich nur noch kriechend fortzubewegen, ist ihre Ausbildung für den Flohzirkus abgeschlossen. $\propto$ Klaus Eschen, Vor den Schranken. Erfahrungen eines linken Anwalts, Kursbuch 40 (1975), S. 104. 
noch als der Moralist kann der Reformer erwarten, daß die zu Recht als strukturkonservativ und innovationsbedürftig analysierte Praxis sich nicht der Analyse gemäß verhält und sich widerstandslos erneuern läßt.

Aber: Die Justizpraktika sind nicht repräsentativ für alle Praktikumserfahrungen, die Rollenprobleme, die Angleichungszwänge und die persönliche Belastung sind dort, bei der Staatsanwaltschaft mehr noch als in der Zivilgerichtsbarkeit, stärker als in den späteren Praktika in der Verwaltung, beim Rechtsanwalt und in der Wahlphase. Ursache mag eine Umstrukturierung der Erwartungshaltungen sein: Man hat gelernt, nicht (mehr) so (zu) hoch zu springen. Nicht rein zufällig werden die Probleme der Praxisausbildung idR am Beispiel der Staatsanwaltschaft abgehandelt. Die Unmittelbarkeit, mit der hier staatliche Macht - und Herrschaftsausübung erfahren werden kann, paart sich mit dem Zwang, ohne entlastende Routine selbstverantwortlich tätig zu werden. Die Situation der Sitzungsvertretung für die Staatsanwaltschaft mit Plädoyer mag die von B. beschriebene kalte Logik des Apparats als das personalverantwortliche Moment von Herrschaft erscheinen lassen.

Modellspezifisch ist für uns dabei aber nur die Tatsache, daß dies in aller Schärfe bewußt wird. Denn die Besonderheit des HM liegt nicht darin, daß in der Praxis überhaupt Angleichungsprozesse stattfinden - eine ihrer wesentlichen Funktionen liegt ja gerade darin, bestimmte Sozialisationseffekte zu erzielen -, sondern in dem zeitlupenartigen, immer wieder durch Besuche in der Universität unterbrochenen Ablauf. Zwar kann man der Chance bewußteren Umganges mit den normativen Erwartungen der Praxis die plausible Erwägung entgegensetzen, daß dauerhafte, wiederholte und stetige Einordnungsleistungen prägenderen Einfluß haben. Die vergleichsweise nur kurzfristige Einbindung in die Praxis eröffnet aber - theoretisch erst die Chance, die real vorhandenen, in den Machtverhältnissen begründeten Restriktionen (gesellschafts-)kritischer Veränderungsbestrebungen der Praxis zu erfahren, ohne in ihnen aufzugehen.

Die Kontrolldichte am Arbeitsplatz dürfte sich von der der Referendare kaum unterscheiden und ebenso ausbilderabhängig sein wie dessen generelle Haltung zum Modell. Die erklärbar ängstlich-abwehrende Haltung gegenüber der Praxis sollte aber nicht dafür herhalten, den eigenen Anteil an der Gestaltung der Praktikumssozialisation aufzulösen in objektivistische Deformationserwartungen.

3. Ein Problem, das im Großen mit Gegenstand der Arbeit der Zentralen Forschungsgruppe zur Juristenausbildung in Mannheim bei der Diskussion um die Vereinheitlichung der Juristenausbildung ist ${ }^{\mathrm{L} \mathrm{T}}$, hat die Studenten mindestens der ersten Jahrgänge wie ein ständiger Schatten begleitet: die Forderung nach Gleichartigkeit, Vergleichbarkeit oder Gleichwertigkeit.

Die Studenten waren auch dann, wenn sie den Reformpostulaten eher skeptisch gegenüberstanden, als $>$ Vertreter des Modells $<$ der besonderen Aufmerksamkeit der Praxis gewiß, von wohlwollender Förderung bis hin zu kaum versteckter Ablehnung war alles vertreten. Ausbildung war der beruhigenden, entspannenden Normalität entzogen. Als Vergleichsmaßstab wurde der für Praktikanten nicht faßbare, durchschnittlich befähigte imaginäre Referendar herangezogen, der - empirisch unwiderlegbar - bei Bedarf aus der Argumentationskiste geholt werden konnte. Mit dem kaum lösbaren Problem belastet, in jeder Handlung die Gleichwertigkeit zu beweisen, sich bewähren, gleichzeitig aber der Erwartung nach etwas Neuem entsprechen zu müssen, waren die Individualisierungsmöglichkeiten begrenzt. Man agierte weniger als Subjekt, mehr als Vertreter einer Gruppe, deren Wert durch den

11 Vgl. Hart, Vereinheitlichung (Fn. I); Joachim Lindner, Wissenschaftliche Legitimation in der juristischen Ausbildungsreform durch Begleitforschung?, KJ I/198I, S. 77-8I. 
Gesamtvergleich mit Referendaren definiert wurde. Ein gesundes Selbstbewußtsein, eine Definition der eigenen Identität unabhängig von den Zuschreibungen zu entwickeln, war unter diesen Bedingungen erschwert, wobei die Probleme der über den zweiten Bildungsweg an die Universität gekommenen Kommilitonen insoweit geringer waren. Distanzierungen vom Modell und seinen Zielen waren die Folge, auch die Ubernahme des Fremdbildes des stets statusniedrigeren, nicht vollwertigen, seinäugigen oder seinbeinigen Rechtspraktikanten.

4. Das Bild der Inferiorität hat sich für die Justizpraktika offensichtlich verfestigt und steht nun in der Welt. Dort sollte es stehenbleiben und nicht weiter beachtet werden. Erfahrungen aus späteren Praktika, insbesondere dem beim Rechtsanwalt, zeigen, daß die Situation an Dramatik noch mehr verliert (auch in den Justizpraktika lautete das Fazit nach den ersten Wochen eher "so schlimm ist es doch gar nicht ${ }^{\mathrm{T}}{ }^{2}$ ), wenn man den Sonderstatus als Rechtspraktikant verdrängt.

Unter diesen Bedingungen ist vom emanzipativen Ziel der kritisch-innovativen Praxisveränderung durch Praktikanten Abschied zu nehmen. Ein derart reduziertes, im übrigen schon auf der Programmebene ambivalentes ${ }^{13}$ Postulat der Integration von Theorie und Praxis wie die Redefinition des gesetzlichen Sozialisationszieles der »Förderung der Fähigkeit des Studenten zu kritischer Mitarbeit und eigenständigem Studium « ( 2 Abs. I EJAG) bedeutet aber nicht die Orientierung am traditionellen Juristen. Für technokratische Vorstellungen wäre diese Perspektive ohnehin dysfunktional, im übrigen stellt sich die Praxis so monolithisch heute nicht mehr dar.

5. Mit der Einphasigkeit der Ausbildung sollte nicht zuletzt auch der Repetitor überflüssig gemacht werden. Damit entfiel von Anbeginn die in traditionellen Fakultäten den wissenschaftlichen Freiraum sichernde institutionalisierte Möglichkeit der Kleinarbeitung des Konflikts zwischen Inhalten und Standards der Ausbildung und den Erwartungen der Praxis. Universitäre Kurse bekommen repetitorenhaften Charakter. Grund dafür ist, daß nun die Vermittlung der für eine erfolgreiche Selbstdarstellung in den Praktika notwendigen materiell- und prozeßrechtlichen Kenntnisse zum alleinigen Problem der Fakultät zu werden scheint.

Hatten die ersten Studienjahrgänge aufgrund der curricularen und personellen Anlaufschwierigkeiten von der Fakultät nur ein begrenztes positiv-rechtliches Wissen vermittelt bekommen, waren sie trotz - oder gerade wegen - der bewußten Defizite doch noch selbstbewußter und bezüglich ihrer >Praxistauglichkeit optimistischer als die dogmatisch weit besser geschulten Kommilitonen nachfolgender Jahrgänge. Zu scheinbar anomischen Zuständen hat die - nicht zuletzt auf Drängen von Studenten erfolgte - Erweiterung des juristischen Lehrangebots als Reaktion auf die Defizitbehauptungen der Praxis, die bis hin zur scheinbar selbstverständlichen Frage nach den Gründen für die eingeschränkte Praxistauglichkeit in den Justizpraktika geht, geführt. Die Bedeutung rechtswissenschaftlicher hard-ware, d. h. vorgeblich praxisrelevanten Wissens, steigt, die Sozialwissenschaften wie die Wissenschaftlichkeit schlechthin sinken im Kurs (sie gehen nicht unter). Gedroht wird mit dem Andrehen der Schraube der Verschulung, zum Wohle der Studenten und des Ruhms der Fakultät. Den Studenten wird so jenes zum Bestehen der Praktika notwendige Maß produktiver juristischer Phantasie, das positive Verhältnis zu den eigenen Lücken und damit das erforderliche Mindestmaß an Selbstbewußtsein ausgetrieben. Der falsche Glaube, die Universität könne und solle hinreichend auf die Praxis

I2 Vgl. K. O., Zum Praktikum bei der Staatsanwaltschaft, in: Paragraphenkotzer, Zeitung der Fachschaft Jura, Heft s/1977.

13 Vgl. Voegeli (Fn. 1), S. $186 \mathrm{ff}$ 
vorbereiten, wird so bestärkt oder es werden jene >Opfer verschulter Studiengänge sichtbar gemacht, die als drop-outs, Verlängerer des Hauptstudiums oder >Verweigerer in den Statistiken auftauchen ${ }^{14}$.

6. Die Folgen der Vorverlegung der Praxisintervention müssen ambivalent eingeschätzt werden. Die frühzeitige Praxisanschauung mag zur Vermeidung von Praxisschocks, die universitäre Sozialisationsversuche vernichten könnten, sehr sinnvoll sein. $>$ Verpraxung des Studiums ${ }^{\text {is }}$ als allzu eilfertige Reaktion der Universität auf teilweise dramatisch klingende Defizitmeldungen aber trägt mit dazu bei, daß die bei Eintritt in das erste Praktikum im Schnitt mindestens drei bis vier Jahre jüngeren Praktikanten schon hier ein deformiertes Selbstbewußtsein haben. Verunsicherung, Scheu oder Angst vor der Praxis können leichter zu jenen Formen der Überanpassung, unreflektierten Abgrenzungsstrategien oder konsequenter Leistungsverweigerung führen, die einer produktiven Umsetzung der Praxiserfahrungen hin zu einer Identitätsbildung in der Rolle als Jurist entgegenstehen.

Wären die Justizpraktika mit ihren Besonderheiten allein prägend für die Ausbildungswirkungen der Praxis, müßte ein Reformer das Scheitern des Modells verkünden. Denn B.'s überzeichnende Beschreibung der Anpassungsprozesse und des damit behaupteten Verzichts auf jegliche Praxisreflexion, auf innovative Bemühungen und auf wissenschaftliche -Entwicklungshilfe für die Praxis enttäuscht die Hoffnung jener, die funktionierende Subsumtionsautomaten, programmiert mit der herrschenden Meinung, vermeiden wollten und als Modellziel den neuen, (herrschafts-)kritischen, innovativen, sozialwissenschaftlichen Erkenntnissen aufgeschlossenen Juristen als Sozialisationserfolg der Ausbildung gesetzt hatten. Eine abschließende Bilanz, welcher Jurist denn nun herauskommt, setzt mindestens aber eine Beachtung der späteren Ausbildungsphasen voraus.

Eine treffendere curriculare Verarbeitung der Praktikumserfahrungen scheint uns der Versuch, unter Abbau des augenblicklich hohen Maßes an bewußter und gewollter Verschulung den universitären Charakter wieder stärker zu betonen ${ }^{16}$.

7. Die Justizpraktika schlagen trotz alledem gewisse Wunden. Sie zu lecken, bietet die Hauptphase II mit dem eigentlichen Modellschwerpunkt »Staats- und Verwaltungsrecht « mehr Gelegenheit, als es nach B.'s Darstellung scheint.

Jedenfalls in unseren Semesterdurchgängen herrschte das Gefühl vor, nach einer wichtigen Erfahrung in einen relativen Freiraum zurückzukehren. Daß die Hälfte des Studiums vorbei war, führte weniger zum erschreckten »dasselbe noch einmal« als zum optimistischen Gefühl, über den Berg zu sein, das "Schlimmste« hinter sich zu haben.

Die Feststellung einer auf den Praktikumserfahrungen beruhenden wachsenden Wissenschaftsfeindlichkeit, die sich »bei vielen Studenten in Theorieskepsis, mangelnder Kritikbereitschaft, mangelnder Bereitschaft, Alternativkonstruktionen zu suchen und sturem Beharren auf der jeweils herrschenden Meinung « ${ }^{17}$ äußere, kann nur getroffen werden, wenn man das sichtbare Studienverhalten an der Fakultät und die durch gründlichere curriculare Vorarbeiten übersteigerten Erwartungen der

I4 Der Leistungsstand hat sich, jedenfalls nach den amtlichen Messungen, gleichwohl nicht gebessert, im Gegenteil (vgl. z. B. Jahresbericht 1979 des Präsidenten des Landesjustizprüfungsamtes (LJPA) bei dem Niedersächsischen Ministerium der Justiz, Nds. Rechtspflege 1980, S. 85 ).

is Vgl. Leibfried (Fn. 1), S. 184 f.; Salge (Fn. I), S. 46.

I6 Nur so besteht die Chance, $\mathrm{z}$ wischen „Berufspraxis sans phrase und „ausbildungsbefreite(r) Hochschulwissenschaft * einen aushaltbaren Vermittlungsmodus zu finden. Wiethölter (Fn. 1), S. 17.

17 Hans-Albrecht Hesse, Kompetenzen der Praktikanten und Bedingungen ihrer Realisierung. Zur Einschätzung des Hannover-Modells nach den ersten Erfahrungen im zivilgerichtlichen Praktikum, RuP 1978, S. 185; vgJ. Salge (Fn. I), S. 46; Jahresbericht (Fn. 14), S. 86. 
Lehrenden zum alleinigen Gradmesser nimmt. Etliche Studenten haben nach den Justizpraktika den Schwerpunkt ihrer Existenz weiter aus der Fakultät für Rechtswissenschaften in andere Lebens-, z. T. auch Studienbereiche verschoben, etwa dadurch, daß sie ein sozialwissenschaftliches Zweitstudium begonnen haben. Andererseits haben an Sozialwissenschaften und kritischer Reflexion uninteressierte Studenten dies deutlicher und offensiver zu erkennen gegeben und damit eventuell die Hoffnungen der Lehrenden enttäuscht, die vorangegangenen Ausbildungsphasen hätten bei allen Studenten den auch von B. so vermißten Hunger nach kritischer Theorie, alternativer Dogmatik und sozialwissenschaftlicher Ausbildung erzeugen können. Daß auch ein noch so ausgeklügeltes Ausbildungsmodell diesen Erfolg nicht produzieren kann, erscheint uns offensichtlich: Kritik als Pflichtprogramm wird notwendig affirmativ.

In einem verschulten Regelstudium anachronistisch anmutende praktizierte Lernfreiheit bestand u. a. in der Teilnahme an und der Mitgestaltung von entsprechenden Lehrangeboten, auch von Vertiefungsveranstaltungen mit projektähnlichem Charakter durch eine relative Minderheit. Unsere Vermutung geht dahin, daß diese Minderheit nicht zuletzt durch den erleichterten Zugang zu derartigen Veranstaltungen größer ist als die an traditionellen Fakultäten. Das Problem war - und ist - nicht, daß Angebote nicht gemacht, Interessen nicht artikuliert oder Wissenschaft nicht betrieben werden könnten, sondern die Motivation der Studenten mehrheitlich auf anderes gerichtet ist. ${ }^{18}$.

8. Der zweite Praktikumsblock - Verwaltung und Rechtsanwalt - hatte dann nur noch wenig von der ,Dramatik der Justizpraktika. Ein Praxis-Schock, wegen der Einführungen, Erkundungen, Vorbereitungen und Rückmeldungen als plötzliches, völlig unerwartetes und unvorhersehbares Ereignis, wie es der Begriff vorgaukelt, schon für die Jusitzpraktika eine nicht zutreffende Beschreibung, war nicht zu spüren. Die Tätigkeit ist $\mathrm{zw}$ ar eine andere, aber eine routinisierte, coolere, gelassenere Haltung gegenüber der Arbeit, den Ausbildern und dem neuen sozialen Umfeld, in das einzuleben notwendig wird, wird zur Regel. Im Grunde kennt man das, was auf einen zukommt, selbst wenn die Einzelheiten genauso offen sind wie bei den ersten Praktika. Den ersten Anpassungseffekten korrespondiert eine erhöhte Resistenzfähigkeit gegenüber weiteren unreflektierten Ubernahmen von Berufsrollen. Bekannt sind auch die einschneidenden Veränderungen des Tagesablaufs, das frühere Aufstehen, die Strukturierung der Tage durch die regelmäßigere Arbeit, die Umstellung von universitärer Wissensakkumulation auf termingebundene Produktion von Entscheidungen und Schriftsätzen; sie treten als Problem wieder auf, sind als zweiter Aufguß aber leichter zu bewältigen. Die Praxis wird als aufgeschlossener und weniger bedrohlich erfahren.

Vor allem die Möglichkeit, sich den Ausbildungsanwalt selbst zu wählen, nimmt dieser Station von Anbeginn vieles vom Charakter der Pflichtstation. Eröffnet wird die Chance, selbst den Ausbildungsprozeß mitzubestimmen, sich einzubringen und, allein schon wegen der beim Anwalt geforderten Selbständigkeit, Selbstbewußtsein zu entwickeln. Schlechte Erfahrungen in der Anwaltsstation wurden nicht als Ausdruck einer allgemein schlechten Praxis genommen, sondern einfach als individuelles Pech bzw. ein Mißgriff bei der Auswahl. Die Interessenbezogenheit anwaltlicher Tätigkeit und damit auch die erleichterten Identifikationsmöglichkeiten,

$18 \mathrm{Ob}$ die spezifischen Ausbildungsbedingungen am HM wesentliches hierzu beitragen, erscheint uns vor dem Hintergrund der Motivationskrise an den geistes- und sozialwissenschaftlichen Fachbereichen mehr als fraglich (vg]. Peter Grottian, Hochschulen wegen mangelnder Motivation geschlossen, päd. extra sozialarbeit Heft 2/1981, S. 42-45). 
die professionelle Ethik der Selbständigkeit und Unabhängigkeit, eventuell auch der nur mittelbare Bezug zur Herrschaft, erklären weiter den insgesamt nicht unangenehmen Verlauf dieser Station. Beschwerend wirkte allenfalls die - nicht unvermeidliche - höhere Auslastung, mitunter auch der Zwang zu mehr Selbständigkeit.

9. Der Endspurt der Ausbildung wie die Verwirklichung des wesentlichen Reformzieles der Spezialisierung wird eingeläutet mit der schwierigen Entscheidung der Wahl des Schwerpunktbereiches. Schwerpunktbildung, an sich schon Realisierung eines Reformpostulats ${ }^{19}$, eröffnet die Chance der Verwissenschaftlichung der Ausbildung, des Einbezuges von Sozialwissenschaften, der vertieften Beschäftigung mit einem Rechtsgebiet wie die Auseinandersetzung mit bestimmten Berufsfeldern und -rollen.

Besondere Bedeutung haben hierbei die Projekte. Relativ selbstbestimmtes, eigenständiges Lernen, die Vertiefung einer Fragestellung auch mit sozialwissenschaftlichen Bezügen sind die positiven Erfahrungen mit den Projekten, gegen curriculare Vereinnahmungsversuche relativ resistente Freiräume. Wer als Student oder Lehrender den vermeintlichen und realen Zwängen eines verschulten Regelstudiums entgehen möchte, hat mit der Projektmöglichkeit eine echte Alternative zum tradierten Lehrbetrieb, um seine Intentionen zu verwirklichen. Stellt man zwangsläufig auftretende Realisierungsschwierigkeiten in Rechnung und euphorischidealistische Zielvorstellungen zurück, bleibt eine erhaltenswerte qualitative Ausbildungsalternative.

Wer das Wahlstudium jedoch allein zur Examensvorbereitung, zum Einpauken juristsicher Dogmatik nutzen will, wird sich auch von noch so geschickt drapierten Sozialisationsversuchen nicht hin zum wissenschaftlich aufgeklärten Juristen drängen lassen.

Uwe Berlit / Horst Dreier

\section{Der einphasige »Rechtshandwerker «}

\section{Vorbemerkung}

Bereits seit $197 \mathrm{I}$ besteht in Augsburg eine einphasige Juristenausbildung, das sog. Münchner Modell. Ein Ausbildungsgang, der den Charakter eines Experiments schon lange verloren hat, der vielmehr relativ festgefahren und relativ unbeeinflußt von der Diskussion über die Zukunft der Juristenausbildung in Augsburg praktiziert wird. Geht man doch davon aus, daß selbst bei einem Wegfall des $₫$ s b Deutsches Richtergesetz (DRiG), der die Möglichkeit von Einphasenmodellen normiert, das Münchner Modell im wesentlichen unverändert weitergeführt werden kann und wird.

Durch die Einführung des $₫ s b$ ins DRiG im September I97I war die gesetzliche Möglichkeit der Einführung von Einphasen-Modellen geschaffen worden. Bayern machte als eines der ersten Bundesländer noch im gleichen Jahr von dieser Möglichkeit Gebrauch. Inzwischen - 1977- wurde auch noch die Juristenausbildung in Bayreuth einphasig organisiert.

r9 Vgl. Alfred Rinken, Einführung in das juristische Studium, München 1977, S. x16; Memorandum des Loccumer Arbeitskreises vom 8. Oktober 1969 zur Reform der Juristenausbildung, in: Loccumer Arbeitskreis (Fn. I), S. 19; Walther Richter, In welcher Weise empfiehlt es sich, die Ausbildung der Juristen zu reformieren, Gutachten $F$ zum 48 . Deutschen Juristentag, München 1970, S. F 110 ff. 
Das Münchner Modell entspricht jedoch von seiner Konzeption her nicht den Anforderungen, die der Loccumer Arbeitskreis an eine reformierte Juristenausbildung gestellt hat. Er wollte die Rechtswissenschaft als Sozialwissenschaft i. S. einer "normativ-empirischen Demokratie-Wissenschaft ${ }^{\prime}{ }^{2}$ verstanden wissen. Organisatorisch sollte dies durch die Integration von theoretischer und praktischer Ausbildung in einem Studiengang, der Neustrukturierung der Ausbildungsinhalte nach Kriterien einer interdisziplinären sozialwissenschaftlichen Aufarbeitung rechtlich relevanter Sozialbereiche und der Reform von Lehr- und Prüfungsformen erreicht werden. Diesen Anforderungen sollte das Münchner Modell "gegenübergestellt «"werden. Es formuliert als Ausbildungsziel: Der angehende Jurist soll während seiner Ausbildung lernen: das Grundwissen des Rechts mit seinen geschichtlichen, philosophischen und sozialwissenschaftlichen Grundlagen, die Methoden seiner wissenschaftlichen Handhabung und praktischen Anwendung, die Stellung des Rechts und seine wissenschaftlichen und politischen Zusammenhänge in der modernen Gesellschaft, die kritische Beurteilung des Rechts und seine Fortentwicklung.

Damit war gesagt, daß sich an den Inhalten der Juristenausbildung schon vom Konzept her nichts Wesentliches ändern sollte. Hauptziel der Reform war und ist die Effektivierung der Ausbildung, yordergründig i. S. einer Verkürzung der Ausbildungszeit, die als wesentlicher Anreiz für ein Studium in Augsburg immer wieder in den Vordergrund gestellt wird. Die hauptsächlichen organisatorischen Elemente zur Erreichung des Ausbildungsziels waren die Integration von Praxisphasen in das Studienprogramm, die Einbeziehung von Nachbarwissenschaften in den Fächerkanon, die Anrechnung der Ergebnisse des r. Examens auf das Abschlußexamen, die Trimesterregelung und ein Kleingruppenkonzept.

\section{Abbau von Reformelementen}

Teile dieser Reformelemente sind im Laufe des Bestehens der Ausbildung bereits wieder abgeschafft worden, so die Trimesterregelung 1978, die Anrechnung der Ergebnisse des ז. Examens 1980. Hinzu kommt noch ein Abbau der schon ursprünglich knapp bemessenen vorlesungsfreien Zeit sowie die geplante Verlängerung der Praktika um 6 Monate, entsprechend den Gesetzesänderungen bezüglich der zweiphasigen Ausbildung. Das Reformmodell wird damit immer weiter auf den Standard der zweistufigen Ausbildung zurückgeführt.

\section{Die Ausbildung im einzelnen}

1. Grundlagen- und Nachbarwissenschaften. Die zentrale Frage der Einbeziehung der Sozialwissenschaften in die Ausbildung ist in Augsburg durch die Aufnahme sog. Grundlagen- bzw. nachbarwissenschaftlicher Vorlesungen gelöst worden. Im Grundstudium werden Vorlesungen in Rechtsgeschichte, Rechtsphilosophie, Rechtssoziologie, Wirtschaftswissenschaften, Psychologie oder Verwaltungslehre

\footnotetext{
I R. Wiethölter, Didaktik und Rechtswissenschaft, in: Loccumer Arbeitskreis (Hrsg.), Neue Juristenausbildung, Neuwied 1970, S. 30.

2 E. Niebler, Die Entstehung des Augsburger Modells der Juristenausbildung, in: Juristische Fakultät der Universität Augsburg (Hrsg.), Die Augsburger Juristenausbildung, Augsburg 1980, S. 24.
} 
angeboten. Im Spezialstudium stehen dann Einführung in die elektronische Datenverarbeitung und Rechtsinformatik auf dem Stundenplan. Dazu kommen dann noch Studienangebote aus den Wahlfachgruppen. Gleichzeitig beginnt jedoch schon im I. Semester die Ausbildung in den juristischen Kernfächern. Die Grundlagen- und Nachbarwissenschaften werden schon formal an den Rand gedrängt. Jeder Student erfährt sehr schnell, was relevant und d.h. primär prüfungsrelevant ist. Die Grundlagen- und Nachbarwissenschaften sind es nicht, hier werden keine Scheine geschrieben und auch im Examen kommen sie höchstens einmal am Rande vor. Dagegen steht die Bedeutung der Kernfächer für das Examen und die rasche Aufeinanderfolge von Scheinen in diesen Gebieten. Betrachtet man dazu noch die Stoffülle der Ausbildung, etwa 26 Pflichtwochenstunden im 6. Semester, wird verständlich, warum nur eine Minderheit der Studenten solche Vorlesungen überhaupt besucht, geschweige denn sich über solch einen Besuch hinaus mit ihnen beschäftigt. Die Rolle dieser Grundlagen- und Nachbarwissenschaften wird dann auch von verschiedenen Seiten kritisch gesehen. Die Ansicht der Studentenvertreter ist eindeutig: "Versagt hat die Augsburger Ausbildung hingegen in der Einbeziehung der Nachbarwissenschaften. « $^{3}$ Auch von seiten des Lehrpersonals werden die Probleme konzidiert. So schreibt der Modellbeobachter Manfred Braun: "Das Angebot an Nachbarwissenschaften stieß demgegenüber auf Skepsis, besonders nach den in das Studium eingeschobenen Praxisphasen, in denen die Studenten vielfach Zweifel über die berufliche Verwertbarkeit nachbarwissenschaftlicher Erkenntnisse, insbesondere der der nichtanalytischen Rechtssoziologie bekommen. « ${ }^{4}$ Das Interesse der Studenten an diesen Fächern schwankt zwischen I $4 \%$ bei Kriminologie und $35 \%$ bei Wirtschaftswissenschaften'. Es finden sich aber auch beschönigende Stimmen. "Was Augsburg seinen Jurastudenten zu den Nachbarbereichen des Rechts und zur Wechselwirkung zwischen dem Recht und der übrigen Wirklichkeit des Gemeinwesens bietet, ist nicht nur dem Studienprogramm nach, sondern auch tatsächlich umfangreicher und besser als die Gerüchte es wahrhaben wollen, die innerhalb und außerhalb Augsburgs umlaufen. ${ }^{6}$

Die Problematik der Nachbarwissenschaften liegt in der Rolle, die ihnen in Augsburg zugedacht wird. Es geht nicht darum, die Rechtswissenschaft als Sozialwissenschaft verständlich werden zu lassen. Rechtssetzung und Rechtsanwendung wird nicht als Ausdruck gesellschaftlicher Verhältnisse bzw. im Wechselspiel mit diesen befindlich gesehen. Das Recht bleibt als abstrakte Wissenschaft erhalten, die Erkenntnisse und Entscheidungen aus sich selbst heraus gewinnt.

Das entspricht natürlich konservativer Bewußtseinshaltung, die so die Funktion des Rechts als systemstabilisierendem Element geschickt vernebelt. Zugeständnisse werden nur insoweit gemacht, als Nachbar- und Grundlagenwissenschaften als solche dargestellt werden, ohne daß Bezüge i. S. einer systemkritischen Hinterfragung des Rechts hergestellt werden.

Der Student, der unvoreingenommen sein Studium in Augsburg beginnt, kann dies zu Ende führen mit einem reichhaltigen Fundus an Kenntnissen in den Gebieten der juristischen Kernfächer, die er in seinem späteren Beruf so bedenkenlos und auch

3 Mögele/Kainz/Wink, Das Augsburger Modell einer einstufigen Juristenausbildung aus studentischer Sicht, in: Juristische Fakultät der Universität Augsburg (Hrsg.), Die Augsburger Juristenausbildung, Augsburg 1980, S. 89.

4 M. Braun, Erste Augsburger Schlußbilanz, JZ 1978, S. 633 ff., 636.

s Examensjahrgang 1979. Abschlußbericht des Modellbeauftragten des Freistaates Bayern an der Universität Augsburg für die einstufige Juristenausbildung, S. 69.

6 D. Suhr, Das Recht und die Nachbarwissenschaften im Augsburger Modell, in: Juristische Fakultät der Universität Augsburg (Hrsg.), Die Augsburger Juristenausbildung, Augsburg 1980, S. 107. 
subjektiv überzeugt von seiner Vorgehensweise anwendet, wie er es im Studium nicht zuletzt an zahlreichen Fallbeispielen gelernt hat.

2. Theorie-Praxis-Bezug. Im Verhältnis von Theorie und Praxis geht es in Augsburg nicht darum, die Praxis am Gericht, am Landratsamt, beim Rechtsanwalt kritisch unter die Lupe zu nehmen. Die Theorie-Praxis-Integration besteht lediglich darin, daß nach 4 Semestern universitärer Ausbildung im Straf- und Zivilrecht ein neunmonatiges Praktikum in diesen Fächern folgt, nach zwei weiteren Semestern Ausbildung an der Uni im öffentlichen Recht folgt dann das 6monatige Verwaltungspraktikum beim Landratsamt. Nach dem 1. Examen folgen noch Praktika beim Rechtsanwalt (drei Monate) und im Spezialfach (drei Monate). Schon die Gewichtung der Praktika in bezug auf das herkömmliche Juristenbild, das von der Tätigkeit am Gericht oder in der Verwaltung ausgeht, macht klar, worauf es in den Praktika ankommt. Schon frühzeitig soll der Student erfahren, was in der Praxis vorgeht, damit er sich entsprechend darauf einstellen und seine Arbeitsschwerpunkte so ausrichten kann, daß sie den späteren Anforderungen in der Praxis genügen. Nicht zuletzt weil die Studenten im ersten Praktikum keinen Bezug zwischen dem, was am Gericht abläuft und den Kenntnissen, die sie u. U. in Grundlagen- und Nachbarwissenschaften erfahren haben, herstellen können, sind sie nach diesem Praktikum noch weniger bereit, sich mit diesen Fächern zu beschäftigen.

Wie gut die Eingliederung in die Praxis gelingt, zeigt die Tatsache, daß die Studenten bei ihrem Eintritt in die Praktika so umfangreiche Kenntnisse in den Studienabschnitten erworben haben, daß sie die anfallenden Arbeiten ohne Verzögerungen bewältigen. ${ }^{7}$ So wird der positive Erfolg der Theorie-Praxis-Integration auch immer wieder herausgestellt. Durch die Hinordnung des theoretischen Studiums auf eine Praxisphase und den Wechsel zwischen theoretischem Studium und praktischer Tätigkeit würden Motivation der Studenten und die Effizienz der Ausbildung insgesamt gesteigert ${ }^{8}$. Auch die Mehrzahl der Studenten sieht die Praktika positiv. So bewerten etwa $55 \%$ den Ausbildungseffekt des Pflichtpraktikum I als sehr gut oder gut und nur $6 \%$ als mangelhaft. ${ }^{9}$

Die unvorbereitete und unreflektierte Ausbildung in der Praxis wird auch nicht etwa in den begleitenden Arbeitsgruppen mit hinterfragenden Akzenten angereichert. Die Arbeitsgruppen - versehen mit kleinlichen Schikanen wie Anwesenheitslisten dienen ausschließlich der Stoffvermittlung. Was in der universitären Ausbildung bereits gelehrt wurde, wird hier vertieft bzw. die Lücken, die noch bestehen, werden geschlossen, vor allem in den Prozeßrechten. Erfahrungen, die die Studenten vor Ort am Gericht etwa machen, finden keinen Eingang in diese praktikumsbegleitenden Arbeitsgruppen. Eine kritische Hinterfragung der Arbeit in der Praxis, geschweige denn eine Einordnung von Gerichten und Verwaltung in einen gesamtgesellschaftlichen Rahmen findet nicht statt. Die Praktika dienen in noch extremerem Maße als die Ausbildung an der Uni der Vermittlung der bestehenden Rechtslehren als System, das es zu akzeptieren und zu reproduzieren gilt, will man sein Studium erfolgreich hinter sich bringen, um sich dann in die bereits erlebte Praxis einordnen zu können.

3. Prüfungssystem. Eine Einschätzung der Ausbildung läßt sich abrunden mit einem Blick auf das Augsburger Prüfungssystem. Als hätte es nie eine Diskussion um Sinn und Unsinn von Blockprüfungen und Prüfungen in Form von Klausuren gegeben.

7 Die einstufige Juristenausbildung in Bayern, eine Information des bayerischen Staatsministeriums der Justiz, München 1978, S. 18.

8 Abschlußbericht des Modellbeauftragten des Freistaates Bayern an der Universität Augsburg für die einstufige Juristenausbildung, S. 20.

9 A. a. O., S. 22 
entscheidet in Augsburg über die Zukunft des Studenten ausschließlich das

Staatsexamen am Ende der Ausbildung. Es besteht aus I 2 Klausuren (4 im Zivilrecht, 2 im Strafrecht, 3 im öffentlichen Recht und 3 im Spezialfach; $\$$ I I 8 JAPO). Dazu kommt eine mündliche Prüfung, die zu $20 \%$ die Gesamtnote bestimmt ( $\$$ I 2 I JAPO).

Die als Reformschritt gepriesene $25 \%$ ige Anrechnung der Note des I. Examens auf die Schlußnote ist bereits wieder abgeschafft worden, um Abweichungen von der zweistufigen Ausbildung zu beseitigen. Hinzu kommen noch 6 Scheine, bestehend aus jeweils einer Hausarbeit und einer Klausur, die im Grundstudium nacheinander bestanden werden müssen, damit die Möglichkeit des Eintritts in den nächsten Ausbildungsabschnitt eröffnet wird, ohne daß ihre Ergebnisse auf die Examensnote irgendeinen Einfluß hätten.

Wichtig zum Bestehen des Examens ist also nur die Fähigkeit, möglichst viel Wissen parat zu haben und zwar in allen Kerngebieten des Rechts, die Fähigkeit dieses Wissen geschickt darzustellen und nicht zuletzt die Fähigkeit, die extreme Prüfungssituation möglichst gut zu bewältigen, die vorhandene Prüfungsangst zu überwinden und zu verdrängen. Es kommt also für den Studenten nicht darauf an, selbständige wissenschaftliche Arbeiten zu erstellen, die Fähigkeit, Zusammenhänge zwischen Gesellschaft und Recht herzustellen oder das Gesellschaftssystem von der Position des Juristen aus kritisch zu hinterfragen, sondern einzig und allein darauf, das Faktenwissen, das im Laufe der Zeit über die Rechtsordnung angesammelt wurde, zu reproduzieren.

Es ist klar, daß solch eine Prüfung das Lern- und Arbeitsverhalten der Studierenden nicht nur unmittelbar vor der Prüfung, sondern während des gesamten Studiums beeinflußt. Es wird einfach so gelernt, damit man im Examen möglichst gut abschneidet, d.h. Konzentration auf die Kernfächer Bürgerliches, Straf- und Verwaltungsrecht, d. h. Anlernen von Wissensbruchstücken, die Prüfungsstoff zu sein pflegen und d.h. weitgehende Außerachtlassung all jener Angebote des Lehrplans, die die Voraussetzung der Examensrelevanz nicht erfüllen, und dies in immer stärkerem Maße, je näher die Examen rücken.

4. Repressionsmöglicbkeiten. Die Effektivität der Ausbildung als Anpassung des Studenten an das herrschende Rechtssystem wird noch verstärkt durch eine Bröckelquote von i. d. R. über $40 \%$, d. h. zum Examen melden sich weniger als $60 \%$ der Studenten, die in einem Jahr das Studium aufgenommen haben (vom Jahrgang r 97 I etwa $55 \%$, vom Jahrgang $\left.197459 \%{ }^{10}\right)$. Wer also bestehen, zumindest das Examen erreichen will, muß sich voll auf die als wichtig angebotenen Lehrinhalte konzentrieren. Hinzu kommen die Möglichkeiten der Zwangsexmatrikulation und der Semestergelder bei Uberschreiten der Regelstudienzeit, die das bayerische Hochschulgesetz für alle seine Studenten als Mittel der Disziplinierung bereit hält. Hinzu kommt für Augsburger Jurastudenten noch die Disziplinierungsmöglichkeiten, die das bayerische Beamtenrecht bietet, dem die Studenten zum erstenmal im Pflichtpraktikum I, als in einem öffentlich-rechtlichen Ausbildungsverhältnis ( $\$ 72$ JAPO) befindlich, unterliegen. Das sind die in Art. 9 I Nr. 2 bayerischem Beamtengesetz geregelten politischen Einstellungsvoraussetzungen für Beamte, konkret das jederzeitige Eintreten für die freiheitlich-demokratische Grundordnung i. S. des Grundgesetzes und der bayerischen Verfassung. Es ist zwar noch kein Fall vorgekommen, in dem ein Student auf Grund dieser Vorschrift nicht ins Praktikum übernommen worden wäre. Angesichts der bayerischen Praxis bei Lehramtsanwär-

10 A. a. O., S. is. 

Juristen in vergleichbaren Fällen entsprechend handeln würde.

\section{Fazit}

Als Fazit ist festzustellen, daß in Augsburg durch die Reform der Juristenausbildung im Münchner Modell die Ausbildung jener Rechtshandwerker effektiviert wurde, die schon in der zweistufigen Ausbildung stattfindet. Die Anforderungen an eine Reform der Juristenausbildung vor allem in inhalt licher Hinsicht, speziell in bezug auf ein Verständnis von Rechtswissenschaft als Sozialwissenschaft, wurden nicht einmal ansatzweise erfüllt.

Thomas Fruth

\section{Referendar-Praxis: Gefühle auf der Titanic}

Saal $57 \mathrm{r}$.

9 Uhr Termin.

Vor dem Saal warten einige Leute. Als ich an der Türe stehen blieb, sehen sie mich neugierig an. Ich nicht. Richter tun das auch nicht. Deshalb heißt es von Richtern, daß sie unparteiisch sind.

Drei Treppen weiter unten wurde ich auf Waffen abgefilzt. So um's Bein rum, im Schritt. Als ich auf den verglasten Sicherheitsbereich zulaufe, unterbrechen die Beamten kurz ihre Unterhaltung. Ich schieße natürlich. Einer kuckt mißtrauisch bis ich um die Ecke verschwinde. Gestern war es schon die dritte Kontrolle. Aufsitzen, hinlegen, absitzen, hinsetzen, vorzeigen, hochheben, lüften, ja lüften. Taschen ausleeren. Machen'se mal gefälligst. Diese alte Ziege mit ihrem Metallfipser. Ich leere aus. Vor sie hin. Ich könne ja wegbleiben, meint sie. Auf solche legen wir keinen Wert. Auf solche! Geuss verhandelt oben im 2. Juni-Prozess und ich bin doch Referendar, Beamter, ich bin doch im Justizdienst. Oder. Der Polizist schlug auf den Anwalt, weil er ihn nicht kannte. Hätte er ihn gekannt, hätte er ihn nicht geschlagen.

Auf dem Gang bewegen sich einige Silberkrawatten. Dünne, phosphorizierende Streifen mit einem steifen Gesicht. Mit Anstand erwachsen geworden. In derartigen Augenblicken des Lebens werde ich ödipal, eine unendlich fortgesetzte Reihe von Vatermorden, wie Fliegen an der Hand. Sein Haar ist zu weiß, als daß er wissen könnte, was in meinem Kopf umgeht. Besser so. Typischer Offiziersschädel, ausrasierter Nacken, bestechend blau. Daneben ein Kollege, sichtlich bemüht, die Pfeife im ausdrucklosen Gesicht festzuhalten. Sie sitzen am Tisch. Der Offizier studiert die Karte. Der andere schneidet ernste Grimassen, fällt aber gegen den Kartätschen-Offizier ab. Auch die Pfeife hält ihn nicht mehr ruhig. Mit dreißig noch bei Muttern. Söhnchen straft jetzt.

Jetzt: Die Tür geht auf und ich laufe quer durch den Saal. Der Staatsanwalt hebt seinen Kopf und mustert mich kurz: ob ich der Angeklagte - nein, ich bin es nicht. Oh Verzeihung. Es ist kurz nach neun.

Ich nicke: guten Morgen.

Auch zu den beiden Schöffen. Der von der Jugendgerichtshilfe ist mir unbekannt. Na ja. 
Ich klammere mich noch an meine Tasche. Der Richter zögert kurz, weiß wohl auch nicht so richtig, dann greife ich zwischen ein paar Finger. Eine elektrisch betriebene Gummihand abgeschüttelt. Die Protokollantin ist wohl sauer, weil ich sie nicht ebenso mit Händeschütteln begrüßt habe. Sie schütteln sich alle. Ein Anwalt braucht dazu - weil sie meist nach den anderen an Gerichtsstelle vertretenen Personen eintreten - schätzungsweise fünf Minuten, bei voller Besetzung, mich inclusive. Distinguierte Personen schütteln manchmal an mir vorbei. Glauben wohl, Referendare holen in den Gerichtspausen Bier und Preßwurst für den Richter. Die mag ich schon gar nicht; ich duelliere ihn zu Tode - wenn die Verhandlung läuft.

Er war im Urlaub. Schön braun. Was soll ich sonst noch sagen.

Alle Beteiligten bitte in Saal 571 eintreten. Lautsprecher. Dieser winzige Satz, zusammen mit anderen winzigen Sätzen, wird täglich ohne grammatikalische Vertauschung gesprochen. Eine gläserne Stimme über dem Korridor, bei dessen Ertönen er aufschreckt. Eine große Tür ist zugesperrt. Als er eintritt, sieht er sich einem weiten Rund gegenüber, das ihn beobachtet. Man müßte einen Furz auf die vollzugsgrauen Flure lassen. Pffft.

Die erste Sache von insgesamt zehn wird verhandelt. Ich beobachte die beiden Schöffen. Sie sitzen aufrecht wie Steiff-Tiere, die Hände stumm auf dem Tisch faltend.

Die Fabel könnte französisch sein: Wie eine Gans versucht, es dem Fuchs gleichzutun, der mit unnachahmlicher Eleganz sich auf seinem Stuhl räkelt, sie dabei aber von aller Welt nur Spott erntet, weil ihre Beine so krumm sind. Und auch noch ihr Schnattern! Es gibt eine Pathologie der Körperhaltung. Nicht daß es anstrengend ist, ruhig zu sitzen. Anders. Es ist anstrengend zu wissen, daß man ruhig sitzt. So wie man Kindern verbietet, in der Nase zu bohren.

Wenigstens steht der Angeklagte auf ebener Erde und nicht eine Treppe tiefer als die Richterbank. Zu den Personalien. Ob denn in der Schule alles glatt ginge. Und sonst auch alles glatt.

Der Richter rutscht etwas nervös auf seinem Stuhl hin und her. Derartige Fragen sind ihm peinlich. Er stellt aber immer dieselben Fragen.

Der Angeklagte nickt nur stumm mit dem Kopf. Der Richter fragt weiter. Erzählen sie mal. Er erzählt, verstockt, sie sind eingeschüchtert, arbeitslos, Lehrling, Schüler, einige bereits im Rückfall, Kinder, die ein schlechtes Gewissen besitzen wegen der geklauten Fußballhandschuhe (er war Torwart in seiner Mannschaft, klar viel Sport), drei sensible Mädchen vom Gymnasium - woran ich das festmache? - vielleicht lesen sie viel, ja, sie lesen viel und tragen andere Kleider, oder an den Lippen; er fragt nach Hobbys, sie hatten Karten für ein Joan Baez Konzert nachgedruckt, wißt ihr, daß man so etwas nicht tun darf. Für Joan Baez würde ich keine Karten nachdrucken, Joan Baez! Obwohl, früher.

Wir suchen nach der sittlichen Verantwortungsreife und Zuchtmitteln. Erziehungsmaßnahmen steht über dem Abschnitt. Die Eltern sind wieder geschieden, oder vom Heim ausgebrochen, die Mutter kaum älter als ihre Tochter, zwei Kinder. Sie hat wieder einen neuen Freund. Kennst du ihn? Sie wendet sich aber nicht um. Ein trotziges hübsches Gesicht. Zum Abschied küßt sie liebevoll ihre kleine Schwester. Sie ist fünfzehn.

So alt wie der Türkenjunge, der einfach mit ein Paar Schuhen davonspaziert war. Hattest du keine Angst? Doch, vor meinem Vater. Er hatte ja das Geld verloren. Wenn das aber alle machen würden, sagt der Staatsanwalt, dann geht doch das Kaufhaus kaputt. Kleiner Türkenjunge, was ich denke, geht wieder niemand etwas an. Die Drogenszene ist versammelt. Am besten gefiel mir der Punk; was ein gelbes Haar, er hatte zehn Gramm Haschplatten verschluckt, im Polizeiprotokoll stand: das 
törnte irre gut; zehn Gramm! Ich muß das Urteil schreiben. Arrest, mangelnde Verantwortungsreife und so' $n$ Quatsch, - schädliche Neigungen -, als würde er zuviel Schokolade essen, oder an einer krankhaften Fallsucht leiden. Gesetz ist Gesetz. Die dumme Bemerkung der Protokollantin über den Rocker. Sie habe nicht mehr länger zusehen können, wie er den Rotz hochgezogen habe. Bis zum Bauchnabel und alle lachten, es war ja so komisch.

Warum sagt der Richter immer dasselbe? Wie z. B.: Und nun hören wir den Vertreter der Jugendgerichtshilfe über dessen Ansicht zum weiteren Verfahren. Ich wies ihn einmal darauf hin. Prompt versprach er sich. Das Stottern war wenigstens glaubwürdig.

Der Vertreter der Jugendgerichtshilfe steht auf. Er plädiert. Ein wohlgesetztes Manual. Es ist ein älterer Herr, der sich seine belegten Brote in die Kantine zum Mittagessen mitbringt. Daß es so billiger sei. Eine Kanne Kaffee dazu. Ein Buch in der Tasche. Und bereits um sieben Uhr geht er mit seiner Tochter schwimmen. Ein alter korrekter Beamter. Der allerletzte Preuße, wie mein Großvater, loyal und treu. Ich gönne mir eine gewisse Sentimentalität.

Während des Redens falten sich die Hände ineinander. Spricht vom kultivierten Elternhaus der Eltern. Er wendet sich ihnen leicht lächelnd zu und nickt betont mit dem Kopf. Seine Fingerspitzen berühren sich leise wie Schäfchen.

Er ist schon 27 Jahre dabei und verbeugt sich. Früher war's am Gericht viel schlimmer, sagt er.

Der Staatsanwalt räuspert sich, steht auf, beugt sich weit nach vorne über seine Akte und beginnt mit seinen Ausführungen. Sachverhaltsschilderung, gut, objektiv: die zur Last gelegte Tat, subjektiv: Vorsatz, das und das. Ein paar dünne Worte zum Strafantrag. Ohne Bewährung. Sieht ihn dabei nicht einmal an. Der Angeklagte meine wohl, er sei ein Märtyrer, als den er versucht, sich uns darzustellen. Arbeit, vernünftiges Leben, das Gericht könne nicht. Kann der Angeklagte? Aufhören. Aufhören. Hör mit dem Gesülze auf. Sie sollten alle die Roben ausziehen. Weil Schwarz so eine vornehme Farbe ist.

Schließlich folgt die Beratung.

Was ich ihm geben soll? Das gleiche wie die Schöffen und damit wie der Richter?

Sag: Scheiße, verlang nach einer rostfreien Guillotine und der Aufhebung des Schwarzfahrverbots. Ich würge. Es bestehen gewisse Schwierigkeiten, Probleme, u.s.w.. Ob ich später Richter werden will. Nein. Wie ich überhaupt zur Strafe stehe. Ihr Wesen ist Repression, sage ich. Der Schöffe redet vom Zuckerschlecken und den Deutschen. Die Frau nickt. Also doch drei Tage. Oder vier. Oder Knast. Zwei Sekunden oder acht Lichtjahre. Zum Schluß drei Freizeitarbeiten und eine Geldbuße an Synanon. Der Richter zuckt selbst mit den Achseln. Manchmal habe ich den Eindruck, daß er auch nicht dahinter steht. Er sieht zum Fenster hinaus.

Urteilsverkündung.

Wir stehen. Dann sitzen wir. Ich ermahne Euch, die Finger da rauszuhalten. Ihr wißt, was das für Folgen hat und die Rechtsmittelbelehrung. Alles in einem Atemzug. Die Sitzung ist geschlossen. Er mußte nochmals darauf aufmerksam machen, daß die Sitzung geschlossen sei. Bitte geh doch.

Der Angeklagte saß unschlüssig auf seinem Stuhl. Das Gericht hat dich gerade verabschiedet oder hast du das nicht gemerkt. Als wäre es eine unerträgliche körperliche Spannung.

Der Vertreter der Jugendgerichtshilfe wird ihn und seine Eltern anweisen. Er verabschiedet sich mit Handschlag. Der Staatsanwalt klappt die Akte zu.

Ein paar mal noch bis gegen Mittag. Dazwischen die Verhandlungspausen. Ich rede 
auch überflüssiges Zeug. Da zwei Termine länger dauern, ist kaum Zeit für das

Mittagessen in der Kantine. Wenigstens Abwechslung.

Nachmittags sind Einzelrichtersachen angesetzt. Der letzte Termin, ein Türke wegen Hausfriedensbruch.

Ich mustere mich mit dem Verteidiger. Er will nicht, daß der Türke neben ihm Platz nimmt, weist mit einer kurzen, schroffen Handbewegung auf den Stuhl vor der Angeklagtenbank. Der Junge duckt sich, ängstlich zusammengekauert, läßt ihn aber nicht aus dem Auge.

Blasiertes Schwein.

Er ist sechzehn, spricht kaum Deutsch. Angeblich sollen sich einige der Türken trotz Hausverbot in einer der Kneipen vom Kudamm-Karrée aufgehalten haben. Belästigung. Ob er wisse, was ein Hausverbot ist. Der Dolmetscher übersetzt.

Ausländerfeindlichkeit bei I,8 Mill. Türken.

Dreißig Minuten dauert nun schon die Verhandlung. Dem Richter wird es auch zuviel. Es ist 15.30 Uhr. Von den Gängen dringen nur noch vereinzelt Geräusche in das Verhandlungszimmer. Eine Tür wird abgeschlossen, das Klappern der Schlüssel, allmählich verlieren sich auch die Schritte in dem Gebäude. Danach ist es still.

Ich höre kaum mehr zu. Wie Honig. Meine Augen sind wie Honig, fallen langsam zu. Ein paar Sonnenstrahlen auf dem Tisch. Es ist warm.

Ein Türke war es, ja ein Türke. Niemand hat mich beobachtet. Der Alte von der Jugendgerichtshilfe döst ebenfalls vor sich hin. Jedenfalls starrt er regungslos auf seine Akte.

Endlich! Dann die Ermahnung SOWAS darf nicht mehr vorkommen. Schon wieder dieser verdammte Satz. Ein müdes Lächeln. Ich sage zu ihm, nur wenn man rede, wäre es möglich, wach zu bleiben. Er nickt abwesend. Auf seine Art hat er es begriffen.

Jetzt laufen wir schweigend den Korridor entlang.

Es erinnert mich an meine ehemalige Schule. Spät am Nachmittag, wenn kein Unterricht mehr war, nur ein altes verlassenes Gebäude, in dem es so seltsam roch und diese merkwürdige Stille. Bis auf die Putzfrauen, die mit ihren Eimern auf den Gängen hantierten.

Niemand der uns begegnet.

Ich versuche mich an die Gesichter der Angeklagten zu erinnern. Umsonst. Es ist eine unendliche Müdigkeit, das Gefühl, drei Tage nicht geschlafen zu haben.

Im Oktober häuft's sich mit den Terminen.

Wir hatten uns, ohne die Hände zu geben, verabschiedet. Ich gondle mit dem Fahrrad mitten im Feierabendverkehr. Es ist beinahe noch heiß, angenehm warm. Als würde ich mit meinem Körper in etwas eintauchen, ertrinken dabei; mich schüttelt's.

Vorne an der Kreuzung hält ein Streifenwagen neben mir. Der eine Bulle mustert mein Fahrrad. Ich stehe direkt an seiner Beifahrerseite und grinse ins Wageninnere. $\mathrm{Na}$ du Affe. Schließlich setzen sie ihre Fahrt im Schrittempo weiter fort.

Und als ich bei der nächsten Ampel noch bei Rot über den Bürgersteig fahre, muß ich laut lachen. Ich fahre jetzt mit meinem Fahrrad bei Rot und auf den Bürgersteig, ja bei Rot und den Knast und das Gericht fahre ich auch zusammen. Mit meinem lächerlichen kleinen Fahrrad fahre ich sie alle zusammen. Tagtäglich schleppe ich eine Bombe in meiner Mappe mit, passiere die vielen Kontrollen und bringe sie wieder mit nach draußen.

Ich tu doch nur so.

Um diese Zeit ist niemand daheim.

Ich geh auf's Clo. Die TAZ. Skandal in Köln, zwei unterschiedlich gehaltene Erklärungen zum 2. Juni Prozess. Teufels B-libi. 
Ich lese die Namen der Unterzeichner. Den kenn' ich auch und die. Natürlich unterschreibt er die hier und nicht die andere. Im Tagesspiegel stand, die Staatsanwaltschaft wolle gegen $6_{3}$ Anwälte wegen standeswidrigen Verhaltens ermitteln. Gerade wegen des Aufrufs. Unehrenhaft.

Einmal die Woche Arbeitsgemeinschaft.

Ich kenne bis heute nur vereinzelt ihre Vornamen. Außer, daß man sich an vieles gewöhnt oder einander freundlich zulächelt. Meist in Begleitung eines Richters, eines Staatsanwaltes oder der Kammer. Es ist das gemeinsame Gefühl, auf der Titanic, das eines verlorenen Rennens oder einfach der falschen Losnummer.

Ich spüle.

Ich würde dämonisieren. Alles.

Angefangen hat es damit, daß ich zur Beweisvernehmung doch etwas entsprechendes anziehen sollte. Nicht viel, keine Umstände. Sie verstehen.

Ein Jackett vielleicht. Vielleicht ein Jackett. Ja? Oder mehr hochgeschlossen. Nein, nein, nicht Krawatte. Rollkragenpulli tut's auch. Er tut's auch! Er trippelte nervös, die Hände in den Hosentaschen im Zimmer auf und ab. Er wolle ja selbst auch nicht. Sie verstehn und bei mir im Haus, daheim.

Er sprach auch nicht direkt vom Anspruch des Staates, sich zu repräsentieren, in geltungsbringenden Formen aufzutreten, oder von alten Zöpfen und die Moderne heutzutage mit ihren überholenden Konventionen. Schon gar nicht von Ungleichzeitigkeit. Nein.

Er sprach vom: Rasieren vielleicht, ha ha ha. Dafür, daß er keinen Arsch in der Hose hatte, konnte ihm nur wirklich kein Vorwurf gemacht werden. Er strich sich mit der flachen Hand über die Nase, als hätte er Schnupfen.

Ich habe auch wieder gelernt, wie wichtig das Einschenken von Kaffee werden kann. Zuerst der Vorsitzende. Nicht besonders erwähnenswert, aber all die Dinge passieren, einfach so. Sind gewollt und passieren. Manchmal gibt es ein weniger davon, ist die Verstrickung geringer, tendenziell und im Verkauf. Nur, hatte ich nicht vom Klassenrecht gesprochen und gehört? Saß ich jetzt im gleichen Boot oder außerhalb, operierend mit einem letztlich diffusen Moralismus ohne Logik außer der einer persönlichen Fügsamkeit, oder war sie nicht immer auch eine verläßliche Moral gewesen? Die Herrschenden, Freiräume erkämpfen - Realabstraktion ohne Realizät? War mit der analytischen Kategorie gleichzeitig eine bestimmte konkrete Realität faßbar und wenn, welche; objektiv, nur ohne Subjekte? Das Problem bliebe dasselbe.

Zuerst wurde gelacht, als ich die Angelegenheit mit dem Rasieren erzählte.

Dann meinte er, nun haben sie Dich auch schon.

Von wegen.

Aber was anderes anziehen wirst Du doch, oder?

$\mathrm{Na}$ ja gut, zieh ich halt ein Jackett an. Was dabei?

Das wird ihm bestimmt gefallen, er lachte, damit arrangierst Du Dich aber.

Entweder steig ich aus oder ich paß mich bis zu einem bestimmten Grade an. Allerdings kommt es darauf an, wie groß der Kompromiß ist. Im Grunde fängt er schon morgens an, genau: Wenn ich durch die Türe gehe und "Guten Morgen« wünsche, anstelle zu sagen: »Halt's Maul.«

Er lachte wieder, richtig, richtig. Du bist ihr Handlanger, stehst auf ihrer Seite. Klassenjustiz.

Es machte ihm Freude.

Das sei wohl doch etwas zuviel, und wir sollten nicht andauernd an unserem Dämon rumbasteln. Von wegen Klassenjustiz. Was denn an dem, was ich tue, großartig politisch sei, hinzugehen und ein paar Stunden abzureißen. Ob dem irgendeine 
Funktion zukomme, neben der, da zu hocken und die mehr oder weniger große Langeweile mit zu tragen. Danach stritten wir uns noch einige Zeit um den Begriff.

Sicher gibt es eine jeweils spezifische Qualität, eine differenzierte Herausbildung und Wahrnehmung von Interessen und auch ihre Nicht-Identitäten. Bloß, in dem auf das Ganze gedachten System liegt die Crux im Detail, in einem hinfälligen Akzeptieren. Bleibe ich authentisch oder die Regel. Wo lege ich mich quer? Als Anwalt zusammen mit Mietern und Arbeitnehmern gegen Vermieter und Arbeitgeber? Deshalb fortschrittlich?

Die Unmittelbarkeit der Bedürfnisse und ihre Einklagbarkeit. Politik in der ersten Person, nicht in der zweiten und auch nicht in der dritten. Ich kenn' das.

Deshalb Personalratspolitik auf Ersatzschauplätzen, weil der Prozeß persönlicher Veränderung eh nicht mehr wieder gut zu machen ist? Fünf Jahre und mehr radikale Politik, die ganze Erfahrung, ein bißchen James-Dean-Gefühl und was bleibt. Doch nur Jugendsünden? Am juristischen Wesen sind schon viele genesen. Dialektik ist, wenn die Konditionierung proportional zum Arbeitsprozeß wächst und ich mir schließlich eine Krawatte ums Bein binde. Ab jetzt Kann-Vorschrift Kollege. Die Methode hat System und umgekehrt.

Ähnliches findet man auch in anderen Berufszweigen. Auf einer Lehrerfete etwa, oder bei Psychologen, die in Supervision vertieft sind. Die Arbeit wird zum Syndrom. Wasser ohne Kohlensäure, dazu noch warm.

Ich fahre fort in meiner Geschichte. Das Miteinander-Auskommen.

So kann sich auf die Dauer - trotz allem - eine Art libidinös besetzter Beziehung entwickeln. Ich registriere, wenn er Schnupfen hat, schlechter Laune ist, aufgeheitert, oder vielleicht müde. Hunger spricht er selber an.

Derart feinfühlig bin ich.

Manchmal wird es mir zuviel. Ich ekele mich vor mir selber. Kaum ein Referendar, dem nicht wenigstens etwas Gebügeltes am Körper haftet. Die Frauen Rock.

Im Augenblick betritt die Kammer für Mietsachen die Kantine. Der Vorsitzende, zwei Beisitzer, die Referendare in den Schlußläufen der Formation. Jetzt setzen sie sich zu Tische. Ja, sie setzen sich richtig hin. Nein, doch noch nicht. A ber jetzt. Doch. Jetzt sitzen sie. Ja sie sitzen. Hurra, sie sitzen. Wie konzentriert er zuhört. Dann tippeln sie wieder weg. Nach dem Mittagessen, auf und davon, wie sie gekommen waren.

Im Vergleich zu den anderen Referendaren hatten wir Glück im Unglück. Zum einen waren wir zu zweit, was hilft. Zum anderen achtete man unsere Erfahrung. Im Ernst. Vielleicht auch wegen unseres Auftretens. Sie schienen uns gegenüber wirklich bemüht, ohne Gemeinheiten, und allein dafür sollte man sie loben. Ebenso in ihrer Fähigkeit Schwächen zu besitzen, wenngleich diese kaum einzugestehen. Es gibt menschliche Verhaltensweisen, bei welchen man sehr genau unterscheiden muß. Innerhalb dessen blieb es ein Gefühl aus Achtung, Ablehnung und Bedauern. Eine Frage der Wahrnehmung: oft genug nicht mehr denn Holzschnitte. Gerade bei Linken: die Moral ist eh auf unserer Seite! Geschichtlich und in ihrer sozialen Totalität. Oder aber als Emanzipationsdefizit begrifflich zumindest ins Reine getragen.

Ich traf sie zwei Minuten nach halb zehn unten am Eingang zur Treppe. Sie hatte Magenschmerzen. Gestern hätte sie geweint, sagte sie.

Eine in den Wahnsinn gehende Kleinlichkeit und Pedanterie. Daß sie nicht beachtet wurde. Einfach kalt. Was, das wissen Sie auch nicht! Was wissen Sie denn überhaupt! Von neuem die Note hingeschleudert. Der beständige Druck. Bis sie schließlich doch kleiner werden. Portables. 
Das wäre also das Glück!

Manche beklagen sich nicht, finden es ginge wohl gut. Ein paar Urteile zu schreiben, Voten, Dezernat bis I $5.00 \mathrm{Uhr}$, kaum länger. Daß die Herrn lieber gestreifte anstatt gepunktete Krawatten trugen, war ihm wichtig.

Vermögensschaden sowie Schmerzensgeld sind mit 60000 .- DM beziffert. Motorradunfall mit kompliziertem Heilungsverlauf-Schädelfraktur -, beide wurden durch den Aufprall beinahe $30 \mathrm{~m}$ durch die Luft geschleudert. Laut Sachverständigengutachten bleibende Folgeschäden. Ungeklärt bleibt nach wie vor der Grad des Verschuldens, da sich die Anstoßgeschwindigkeit der Fahrzeuge nicht einwandfrei rekonstruieren läßt.

Es liegen zwei voneinander abweichende technische Gutachten vor.

Die Anwälte lehnen den Vergleichsvorschlag ab.

Im Ergebnis dennoch Schadensteilung.

Sie werden Berufung einlegen.

Der eine Beisitzer spielt sich während der Beratungspause auf, bis ihn der Vorsitzende unterbricht: Das sei nicht wichtig. Seine Unterlippe schiebt sich beleidigt nach vorne. Man hatte ihm seinen Lolli weggenommen. Für die Dauer eines Augenblicks, danach hat er sich wieder aufgerichtet.

Wir stehen im Kreis um den Tisch und laufen wie Hühner hin und her.

Die Tür öffnet sich, die Anwälte betreten den Saal. Die Art der Verbeugung: Kein bloßes Grüßen oder nachlässiges Kopfnicken. Es ist Bezeugung, eine kurze untergebene Leidenschaft, innere Haltung und Devotion gegenüber dem Gericht. Hiernach ist normales sich Setzen beinahe nicht mehr möglich. Vor allem, wenn sie jünger sind. Die Spannung wird zur Pose. Sie warten auf der Bank, blättern in ihren Akten. Wenn sie vor der Richterbank sitzen, blättern sie wieder in ihren Akten. Machen die Finger naß und blättern. Oder rücken die Krawatte zurecht. Sie sitzen eine Stufe tiefer als wir.

In einer Untersuchung über die Novellierung der Zivilprozeßordnung wurde die Funktion der Gerichte als Dienstleistungsbetrieb der großen Gesellschaften benannt. Anwälte, die nicht ihre Klienten kennen und Gerichte, die mathematisch verfahren. Die dünne Luft der Parteien. Ich sehe kaum mehr beteiligte Personen, nur Antragsteller und Antragsgegner. Schriftsätze, in denen bei einem schweren Unfall lapidar von einer Trübung der Lebensfreude gesprochen wird, um auf den Kostensatz zu schielen, ohne sich im geringsten von irgendwelchen persönlichen Konsequenzen betroffen zu sehen. Beinbrüche, zerquetschte Leber, verkürzte Gliedmaßen, Amputationen. Geschäft ist Geschäft.

Wirkliche Streitigkeiten nur dann, wenn sie sich gegenseitig für dumm halten und das auch zu erkennen geben.

Subtil natürlich.

Vor mir eine Dickwurst, mit Brille. Aufgeblasen, die langsame, überlegt vorgenommene Handbewegung, mit der er sie abnimmt und vor sich hinlegt. Eine Studie. Er spielt jetzt: Nach hinten ausgebreitet zurückgelehnt.

Hohes Gericht.

Unaufhaltsam und unangenehm. Dazwischen Kunstpausen. Sogar mich lächelt er noch an.

Die meisten dürften Tennis spielen.

Bis auf den Rest. Sie unterhalten sich über Kollegen. Über den. Daß er einen unangenehmen Körpergeruch habe, gewissermaßen stinkt, sagt er.

Er ist Richter einer anderen Kammer und hat heute die Vereidigung eines Beisitzers übernommen. Wir sitzen in einer Pizzeria zu Mittag und plaudern.

Unangenehm korrekter Mensch. Wahrscheinlich anal. Sie hätten alle 'mal scheißen 
sollen, dann wäre Ihnen der reinliche Fleiß verloren gegangen. Dieser erscheint in jeglicher Form: Als ausgeprägter Infantilismus - auch den gibt es - wie in seiner aggressiv-dominanten Art. Phallokratisch paßt nicht bei Frauen.

Sie sah phantastisch gut aus. Daß die bei Frauen immer daran denken. Zumindest als erstes erwähnen.

Morgen muß ich einen Aktenvortrag halten. Ausgerechnet morgen. Was ist morgen?

Die alten Lords im Metropol. Ach Gott, ach Gott. Pflanzen könnte ich mir 'mal wieder kaufen. Wozu habe ich denn mein Referendarsgehalt? Bin doch kein Lehrer und kauf bei Ikea Elche. Trödel, gut, aber Ikea?

Trödel ist wenigstens politisch.

Ich trage noch ein paar Akten von der Registratur nach oben.

Es gibt nichts mehr. Ob ich denn auch ins Tempodrom ginge? Die Luft in dem kleinen Zimmer riecht muffig. Es wird nicht gelüftet.

Eckart Wähner 